The Astrophysical Journal, 660:1232-1245, 2007 May 10

(C) 2007. The American Astronomical Society. All rights reserved. Printed in U.S.A.

\title{
GRAVITATIONAL RUNAWAY AND TURBULENCE DRIVING IN STAR-GAS GALACTIC DISKS
}

\author{
Woong-Tae Kim ${ }^{1}$ and Eve C. Ostriker ${ }^{2}$ \\ Received 2006 June 1; accepted 2007 January 24
}

\begin{abstract}
Galactic disks consist of both stars and gas. The gas is more dynamically responsive than the stars, and strongly nonlinear structures and velocities can develop in the interstellar medium even while stellar surface density perturbations remain fractionally small. Yet, the stellar component still significantly influences the gas. We use two-dimensional numerical simulations to explore formation of bound condensations and turbulence generation in the gas of two-component galactic disks. We represent the stars with collisionless particles, follow their orbits using a particle-mesh method, and treat the gas as an isothermal, unmagnetized fluid. The two components interact through a combined gravitational potential that accounts for the distinct vertical thickness of each disk. Using stellar parameters typical of mid-disk conditions, we find that models with gaseous Toomre parameter $Q_{g}<Q_{g \text {.crit }} \sim 1.4$ experience gravitational runaway and eventually form bound condensations. This $Q_{g \text {,crit }}$ value is nearly the same as previously found for razor-thin, gasonly models, indicating that the destabilizing effect of "live" stars offsets the reduced self-gravity of thick disks. This result is also consistent with empirical studies showing that star formation is suppressed when $Q_{g} \gtrsim 1-2$. The bound gaseous structures that form have mass $6 \times 10^{7} M_{\odot}$ each, representing superclouds that would subsequently fragment into GMCs. Self-gravity and sheared rotation also interact to drive turbulence in the gas when $Q_{g} \gtrsim Q_{g \text {,crit. }}$ This turbulence is anisotropic, with more power in sheared than compressive motions. The gaseous velocity dispersion is $\sim 0.6$ times the thermal speed when $Q_{g} \approx Q_{g \text {,crit }}$. This suggests that gravity is important in driving interstellar turbulence in many spiral galaxies, since the low efficiency of star formation naturally leads to a state of marginal instability.
\end{abstract}

Subject headings: galaxies: ISM — galaxies: structure — instabilities — ISM: kinematics and dynamics solar neighborhood — stars: formation — stars: kinematics

\section{INTRODUCTION}

Large-scale galactic disk evolution is governed by the interaction between self-gravity and other reinforcing and/or countervailing forces. Some of these forces are associated with sheared galactocentric rotation, and others are associated with random velocities (thermal and turbulent) of the gas and stars. Under certain circumstances, gravitational instabilities may grow and radically alter the disk structure as they develop nonlinearly. This is believed to be how giant molecular clouds (GMCs), and subsequently starforming $\mathrm{H}$ II regions, originate. Under other circumstances, the overall disk morphology is not changed, but self-gravitating modes grow and interact in such a way that significant energy is transferred from ordered to disordered forms. This "disk heating" may in turn affect the system's subsequent susceptibility to selfgravitating instabilities.

Because the gas component is much cooler than that of the stars and because gas can radiate energy as it is nonlinearly compressed, the effects of self-gravitating instabilities are much more pronounced in the interstellar medium (ISM) than in the stellar disk. The stellar component can nevertheless be quite important to the initial growth of self-gravitating modes (or waves), because it contains so much of the disk's mass $(\sim 75 \%-90 \%$, compared to $\sim 10 \%-25 \%$ in gas). While the stars are essentially collisionless, most previous work on gravitational instability in two-component disks has treated both stars and gas as two isothermal fluids with different sound speeds. This approach was taken by Jog \& Solomon (1984a, 1984b), Elmegreen (1995b), and Jog (1996) in studying axisymmetric self-gravitating modes, and by Jog (1992) and Bertin \& Romeo (1988) in studying nonaxisymmetric self-gravitating

\footnotetext{
${ }^{1}$ Department of Physics and Astronomy, Frontier Physics Research Division, Seoul National University, Seoul 151-742, South Korea.

2 Department of Astronomy, University of Maryland, College Park, MD 20742.
}

modes for combined star/gas disks (see also Bertin \& Lin 1996). Adopting a full kinetic treatment for the stellar component in twocomponent disks, on the other hand, Lin et al. (1969) and Rafikov (2001) used linear perturbation theory to derive dispersion relations for nonaxisymmetric WKB waves and axisymmetric modes, respectively. These linear theory analyses cannot capture, however, the ultimate outcomes - including GMC formation and turbulent driving - which result when localized, self-gravitating structures grow; quantifying this development requires nonlinear numerical simulations.

The main class of self-gravitating instability that develops under conditions of strong sheared rotation (in outer galaxies, away from spiral arms) is the swing amplifier (Goldreich \& Lynden-Bell 1965b; Julian \& Toomre 1966; Toomre 1981). In Kim \& Ostriker (2001, hereafter Paper I), we identified the basic requirements for swing to occur and reported on the results of extensive numerical simulations of swing amplification in gaseous disks with or without magnetic fields. Although in linear theory the amplification magnitude varies continuously with respect to the Toomre parameter $Q_{g}$ (see eq. [6] for definition), Paper I showed that nonlinear interactions among swing-amplified density filaments or wavelets lead to a threshold phenomenon. Disks with $Q_{g}>Q_{g \text {,crit }}$ remain stable, while disks with $Q_{g}<Q_{g \text {,crit }}$ experience gravitational runaway, forming bound condensations. For the razor-thin disk models of Paper I, we found that $Q_{g \text {,crit }} \sim 1.2-1.4$, with the largest value resulting from models with subthermal magnetic fields. Given the similarity between the numerically obtained $Q_{g \text {,crit }}$ values and observationally inferred thresholds for active star formation in external galaxies (Quirk 1972; Kennicutt 1989; Caldwell et al. 1991; Martin \& Kennicutt 2001; Wong \& Blitz 2002), Paper I supported the notion that self-gravitating instabilities define the star formation "edges" of disks.

While successfully demonstrating the nonlinear threshold behavior of the swing amplifier and quantitatively yielding good 
agreement with observations, the models considered in Paper I suffered from two important drawbacks. First, by assuming infinitesimally thin disks they overestimated self-gravity, which tends to overestimate $Q_{g \text {,crit }}$; second, they did not allow for a dynamically active stellar disk, which tends to underestimate $Q_{g, \text { crit }}$. Subsequent simulations of pure-gas disks in three dimensions confirmed the basic nonlinear threshold behavior, but also showed that for realistic disk temperatures the nonzero thickness of the disk can indeed significantly lower the value of $Q_{g \text {,crit }}$ to $<1$ (Kim et al. 2002).

In this paper we address the second limitation of Paper I by studying nonlinear evolution of gravitational instabilities in $t w o-$ component galactic disks consisting of both stars and gas. We distribute collisionless particles to represent stars and follow their orbits using a direct $N$-body method, while adopting a hydrodynamic approach for gas. The two components are allowed to interact with each other through the combined gravitational potential (calculated on a single mesh). We also allow for nonzero (and differing) vertical thickness of both stellar and gaseous disks in solving the Poisson equation for each component.

Given that finite-thickness effects tend to reduce $Q_{g, \text { crit }}$ values below observed star formation thresholds in gas-only models, a compelling question is whether allowing for an active stellar disk compensates for this effect. Thus, one of our primary objectives is to quantify the critical $Q_{g}$ value for gravitational runaway when both a live stellar component and a thick disk are explicitly considered. A second important goal is to understand what happens when $Q_{g}$ is sufficiently large that gravitational runaway does not occur. In particular, the large-scale flows that are driven when gravity is strong (but not too strong) may be a key to powering turbulence within the disk.

Many processes may contribute to turbulence in the diffuse gas. In addition to self-gravitating instabilities, other candidate mechanisms that have been proposed include thermal instabilities, the Parker and magnetorotational instabilities, dynamical instabilities in spiral shocks, stellar winds, and expanding stellar $\mathrm{H}$ II regions and supernova blast waves (see Mac Low \& Klessen 2004; Elmegreen \& Scalo 2004 for recent reviews). Traditionally, stellar processes have been presumed to be the dominant turbulence drivers, but the lack of observed spatial correlation between velocity dispersions of $\mathrm{H}$ I gas and regions of high-mass star formation (Dickey et al. 1990; van Zee \& Bryant 1999) suggests that other sources must be important as well. In particular, Elmegreen (2002) and Elmegreen et al. (2003) have suggested that the swing amplifier generates large-scale motions that cascade to produce turbulence and structure on small scales as well as large scales. Initial numerical studies of this process have been pursued by Wada et al. (2002), who performed two-dimensional, thin-disk galactic simulations with ISM cooling. They found that a cloudy medium develops, with a velocity dispersion $\sigma_{v} \sim 2-5 \mathrm{~km} \mathrm{~s}^{-1}$. On the other hand, Kim et al. (2003) found via three-dimensional isothermal simulations, with sound speed $c_{g}=7 \mathrm{~km} \mathrm{~s}^{-1}$ and $Q_{g} \gtrsim 1.7$, that turbulence was driven to levels less than $1 \mathrm{~km} \mathrm{~s}^{-1}$. Based on just these few models, it has not yet been clear how important "swingdriven" turbulence is more generally.

In the present work we determine how the turbulent velocity amplitude of the gas, $\sigma_{v}$, varies as a function of $Q_{g}$ and also assess how the presence of a live stellar component affects $\sigma_{v}$. By running a number of models with a range of particle numbers and also performing control models with "passively evolving" stars, we are able to subtract out the contribution to $\sigma_{v}$ due to Poisson noise in the particle distribution. We show that quite high particle resolution is in fact necessary for accurate determination of $\sigma_{v}$ in the case when $Q_{g}$ is near $Q_{g \text {,crit }}$; this is of practical importance because real disks naturally evolve toward near-critical states.
This paper is organized as follows. In $\S 2$ we present the basic equations we solve for gas and stars, introduce "thick-disk" gravity, and describe our model parameters. In $\S 3$ we revisit the linear theory for axisymmetric gravitational instability in two-component disks with an emphasis on the stabilizing effect of nonzero disk thickness (omitted in previous studies). In $\S 4$ we describe numerical methods we employ for our simulations and present results of code tests. We demonstrate that large Poisson noise in the stellar particle distribution can lead to spurious results for $N$-body systems that are nearly or marginally gravitationally unstable. In $\S 5$ we report on our simulations of two-component disks. We present numerical results on $Q_{g}$ thresholds for (nonaxisymmetric) gravitational runaway and quantify the overall level and spectral properties of gravity-driven turbulence when $Q_{g} \gtrsim Q_{g \text {,crit }}$. Finally, we summarize our results and discuss their astronomical implications in $\S 6$.

\section{EQUATIONS AND MODEL PARAMETERS}

\subsection{Basic Equations and Thick-Disk Gravity}

In this paper we investigate the dynamics of local galactic disks composed of gas and stars. We follow the evolution of the gas by solving the hydrodynamic equations, while employing an $N$-body method for the stars. The gas is assumed to be isothermal and unmagnetized, and we assume that all physical variables other than the gravitational potential are independent of the vertical height. As in Paper I, we consider a patch of the disk orbiting the galaxy with a fixed angular frequency $\Omega_{0}=\Omega_{0} \hat{z}$, and set up a local Cartesian frame with $x$ and $y$ referring to radial and azimuthal coordinates, respectively. We consider a simulation box with size $L \times$ $L$. The equilibrium background velocity arising from galactic rotation relative to the center of the box at $x=y=0$ is given by $\boldsymbol{v}_{0}=-q \Omega_{0} x \hat{\boldsymbol{y}}$, where $q \equiv-d \ln \Omega / d \ln R$ denotes the dimensionless local shear rate. The basic dynamical equations we solve for the gaseous part in the local frame are identical to those presented in Paper I, except that we adopt here a "thick-disk" gravitational kernel in solving the Poisson equation (see below).

To follow the evolution of the stellar component, we use collisionless particles. Restricting particle motions to be in-plane, the shearing sheet equations of motion relative to the center of the box are given by

$$
\ddot{\boldsymbol{r}}=2 q \Omega_{0}^{2} x \hat{\boldsymbol{x}}-2 \boldsymbol{\Omega}_{0} \times \dot{\boldsymbol{r}}-\nabla\left(\Phi_{g}+\Phi_{s}\right),
$$

where $\boldsymbol{r}=x \hat{\boldsymbol{x}}+y \hat{\boldsymbol{y}}$ is the position vector of a particle, the dots denote derivatives with respect to time (e.g., Julian \& Toomre 1966; Wisdom \& Tremaine 1988), and $\Phi_{g}$ and $\Phi_{s}$ are the gravitational potentials from gas and stars, respectively. The spatial distribution of the particles at any given time will give the stellar surface density $\Sigma_{s}$, which in turn yields $\Phi_{s}$ via

$$
\nabla^{2} \Phi_{s}=4 \pi G \Sigma_{s} h_{s}(z)
$$

where $h_{s}(z)$ represents the vertical distribution of the stellar density and satisfies the normalization condition $\int_{-\infty}^{\infty} h_{s}(z) d z=1$. The Poisson equation for the gas takes the same form as equation (2) with subscripts changed appropriately.

Studies of disk dynamics often assume $h(z)=\delta(z)$ for simplicity. This thin-disk approximation would be valid as long as perturbations of interest do not critically rely on the vertical dimension and their wavelengths $\lambda$ are much larger than the disk scale height $H$. For waves with $\lambda$ approaching $2 \pi H$, however, the thin-disk approximation overestimates self-gravity at the disk midplane. This can be particularly severe in a two-component system 
in which the characteristic length scales and disk scale heights for gas and stars are quite different from each other. For instance, the critical wavelengths for axisymmetric gravitational instability under solar neighborhood conditions are $\sim 10 \mathrm{kpc}$ for stars (e.g., Binney \& Tremaine 1987) and $\sim 1$ kpc for gas (e.g., Elmegreen 1995a; Paper I). The stellar scale height $H_{s} \sim 330 \mathrm{pc}$ (Chen et al. 2001) is nonnegligible compared with the wavelength of the most vulnerable modes in the combined system. Moreover, as perturbations grow via swing amplification they form thin overdense filaments with widths comparable to the gas disk's scale height (Paper I; see also $\S 5.1$ ), so that the thin-disk gravity may potentially lead to spurious fragmentation. It is thus desirable to allow for finite disk thickness in solving equation (2).

Suppose that $h_{s}(z)$ does not vary with time and that $\Sigma_{s}$ is periodic in $x$ and $y$ (or linear combinations of coordinates; see $\S 4.1$ ). Using the Green's function method, one can show that equation (2) yields the solution at $z=0$ (omitting the subscript $s$ )

$$
\Phi(\boldsymbol{k})=-\frac{2 \pi G \Sigma(\boldsymbol{k})}{|\boldsymbol{k}|} \int_{-\infty}^{\infty} h\left(z^{\prime}\right) e^{-\left|\boldsymbol{k} z^{\prime}\right|} d z^{\prime},
$$

where $\Phi(\boldsymbol{k})$ and $\Sigma(\boldsymbol{k})$ refer to the Fourier components with wavevector $\boldsymbol{k}$. When $h(z)=e^{-|z| / H} /(2 H)$, equation (3) simply becomes

$$
\Phi(\boldsymbol{k})=-\frac{2 \pi G \Sigma(\boldsymbol{k})}{|\boldsymbol{k}|(1+|\boldsymbol{k}| H)},
$$

which we refer to as "thick-disk" gravity, as opposed to thin-disk gravity, for which $H$ is set equal to zero. This result was obtained through a different technique by Elmegreen (1987) and has been shown to be quite a good approximation for the reduction of selfgravity in disks with various density profiles (e.g., Romeo 1992; Kim et al. 2002). By direct numeral integration, one can see that equation (4) slightly underestimates the real potential for purely self-gravitating disks, but not more than $14 \%{ }^{3}$ Kim \& Ostriker (2006) further found that when equation (4) is used to calculate self-gravity, simulations of gas flow across two-dimensional spiral shocks produce results very similar to fully three-dimensional models. In what follows, we shall use equation (4) to compute the self-gravity of gas and stars with scale heights $H_{g}$ and $H_{s}$, respectively.

\subsection{Model Parameters}

As initial models we consider a composite disk of gas and stars with uniform surface densities $\Sigma_{g 0}$ and $\Sigma_{s 0}$. The gaseous disk is set to remain isothermal throughout the entire evolution with an effective speed of sound $c_{g}=7 \mathrm{~km} \mathrm{~s}^{-1}$, corresponding to a mean Galactic thermal pressure $P / k \sim 2000-4000 \mathrm{~K} \mathrm{~cm}^{-3}$ (Heiles 2001) and mean midplane density $n_{\mathrm{H}} \sim 0.6 \mathrm{~cm}^{-3}$ (Dickey \& Lockman 1990), similar to solar neighborhood values. For the stellar disk, we initially distribute particles according to the Schwarzschild distribution function

$$
f\left(v_{x}, v_{y}\right)=\frac{\Sigma_{s 0}}{2 \pi \sigma_{s, x} \sigma_{s, y}} \exp \left[-\frac{v_{x}^{2}}{2 \sigma_{s, x}^{2}}-\frac{\left(v_{y}-v_{0, y}\right)^{2}}{2 \sigma_{s, y}^{2}}\right],
$$

where $\sigma_{s, x}$ and $\sigma_{s, y}$ are the $x$ - and $y$-components of the particle velocity dispersion, respectively. The values of $\sigma_{s, x}$ and $\sigma_{s, y}$ will vary over time as the particles respond to the perturbed gravitational

\footnotetext{
3 When eq. (4) is used to obtain an axisymmetric dispersion relation for a disk with $H_{g}=c_{g}^{2} /\left(\pi G \Sigma_{g}\right)$ (i.e., $H_{g}$ determined by gas self-gravity alone), the resulting $Q_{\text {crit }}=0.65$ is very close to the value $Q_{\text {crit }}=0.68$ obtained by Goldreich \& Lynden-Bell (1965a) as an exact criterion for axisymmetric instability.
}

potential. Note that $\Sigma_{s 0}=\int f d v_{x} d v_{y}$ and $\sigma_{s, y} / \sigma_{s, x}=(1-q / 2)^{1 / 2}$ in equilibrium. We initially adopt $\sigma_{s, x}=30 \mathrm{~km} \mathrm{~s}^{-1}$ and $\sigma_{s, y}=$ $2^{-1 / 2} \sigma_{s, x}$, corresponding to solar neighborhood conditions with a flat $(q=1)$ rotation curve (Binney \& Tremaine 1987).

When written in dimensionless form, the basic dynamical equations are characterized by the parameters

$$
\begin{gathered}
Q_{g}=\frac{\kappa_{0} c_{g}}{\pi G \Sigma_{g 0}}, \quad Q_{s}=\frac{\kappa_{0} \sigma_{s, x}}{3.36 G \Sigma_{s 0}}, \\
n_{\mathrm{J}}=\frac{G \Sigma_{g 0} L}{c_{g}^{2}}, \quad X=\frac{\kappa_{0}^{2} L}{4 \pi^{2} G \Sigma_{s 0}},
\end{gathered}
$$

together with $H_{g} / L$ and $H_{s} / L$. The Toomre $Q$ parameters defined by equation (6) give the surface densities relative to the critical values at $Q_{g}=Q_{s}=1$ for axisymmetric gravitational instability in a razor-thin, gas-only or star-only disk ( Toomre 1964; Binney $\&$ Tremaine 1987). In equations $(7)$, the $n_{\mathrm{J}}(X)$ parameter is the ratio of the simulation box size $L$ to the critical wavelength $\lambda_{g \text {,crit }}=$ $c_{g}^{2} / G \Sigma_{g 0}\left(\lambda_{s, \text { crit }}=4 \pi^{2} G \Sigma_{s 0} / \kappa_{0}^{2}\right)$ for axisymmetric gravitational instability in a razor-thin, nonrotating gaseous (rotating, cold stellar) disk.

Since the parameter space is very large and since we are primarily interested in the dynamical evolution of the gaseous component in combined disks, we vary only $Q_{g}$ (or equivalently $\Sigma_{g 0}$ ) and fix all the other disk properties. For the stellar part, we adopt solar neighborhood values $\kappa_{0}=36 \mathrm{~km} \mathrm{~s}^{-1} \mathrm{kpc}^{-1}$ (Binney \& Tremaine 1987), $\Sigma_{s 0}=35 M_{\odot} \mathrm{pc}^{-2}$ (Kuijken \& Gilmore 1989), and $H_{s}=330 \mathrm{pc}$ (Chen et al. 2001; Karaali et al. 2004). This gives $Q_{s}=2.1$, so that in our model disks the stellar component alone, even in the razorthin limit, would be immune to axisymmetric instability. We adopt $H_{g}=170 \mathrm{pc}$ for the effective scale height of the local ISM (e.g., Boulares \& Cox 1990). For the box size, we adopt $X=2$ or $L=$ $9.2 \mathrm{kpc}$, which is large enough to resolve the most susceptible modes of the swing amplifier in stellar disks ( Toomre 1981); test simulations we have performed confirm that results are insensitive to $X$ as long as $X \geq 2$.

Our fiducial model has $Q_{g}=1.4$ corresponding to $\Sigma_{g 0}=$ $13 M_{\odot} \mathrm{pc}^{-2}$ for the gas disk (e.g., Holmberg \& Flynn 2000), but we also allow for various values of $Q_{g}$ in the range of $1-3$. These models will allow us to determine the critical $Q_{g}$ value for gravitational runaway and to study the characteristics and level of turbulence driven by self-gravity when $Q_{g} \gtrsim Q_{g \text {,crit }}$. Since equations (6) and (7) give $n_{\mathrm{J}}=3.7 X \sigma_{s, x} /\left(c_{g} Q_{g} Q_{s}\right) \sim 15 / Q_{g}$ for the adopted set of parameters, we see that our simulation box is large enough to contain at least five Jeans wavelengths of the gaseous medium. We define the orbital period $t_{\text {orb }} \equiv 2 \pi / \Omega_{0}=2.4 \times$ $10^{8} \mathrm{yr}\left(\Omega_{0} / 26 \mathrm{~km} \mathrm{~s}^{-1} \mathrm{kpc}^{-1}\right)^{-1}$ and use it as the time unit in our presentation.

\section{AXISYMMETRIC STABILITY}

The gravitational stability of two-component disks to axisymmetric perturbations was first analyzed by Jog \& Solomon (1984a), Romeo (1992), Elmegreen (1995b), and Jog (1996), who treated the stellar disk as an isothermal fluid. While a fluid description of stellar particles is a good approximation for a range of wavenumbers in disks near the threshold, it generally fails when disks are further from instability or when the wavelengths of perturbations are small compared to the epicyclic excursions of stars (e.g., Binney \& Tremaine 1987). Rafikov (2001) instead used a collisionless description of the stellar population and derived a dispersion relation for axisymmetric waves in star-gas disks assuming that the disk is razor-thin. In this section we revisit axisymmetric instability with a particular emphasis on the effect of nonzero disk thickness. 
The results of this section will be used to check our numerical technique in $\S 4.2$.

We refer the reader to Rafikov (2001) for a detailed derivation leading to the thin-disk dispersion relation for two-component disks. We follow the same steps, except we use thick-disk equation (4) for the potential and density pairs in place of Rafikov's equation (14). The resulting dispersion relation for axisymmetric modes in a two-component disk of finite vertical extent reads

$$
\begin{aligned}
& \frac{2 \pi G k \Sigma_{g 0}}{\left(\kappa^{2}+k^{2} c_{g}^{2}-\omega^{2}\right)\left(1+k H_{g}\right)} \\
& +\frac{2 \pi G k \Sigma_{s 0} \mathcal{F}\left(\omega / \kappa, k^{2} \sigma_{s, x}^{2} / \kappa^{2}\right)}{\left(\kappa^{2}-\omega^{2}\right)\left(1+k H_{s}\right)}=1,
\end{aligned}
$$

where $\omega$ and $k$ are the frequency and radial wavenumber of perturbations, respectively, and $\mathcal{F}$ is the stellar "reduction factor" defined by equation (6-45) of Binney \& Tremaine (1987), which accounts for the effects of the stellar velocity dispersion. A similar relation was presented by Romeo (1992), who introduced a single effective scale height for the combined disk instead of using different heights $H_{g}$ and $H_{s}$ for each component. In the limit of $H_{g}=H_{s}=0$, equation (8) recovers the thin-disk dispersion relation of Rafikov (2001). Note that although a combined thick disk can be mapped to a razor-thin counterpart with lower surface density, the density reduction factors of the gaseous and stellar components differ from each other and cannot be determined a priori, since they depend on the perturbation wavenumber.

We determine the marginal stability condition by solving equation (8) with $\omega^{2}=0$ and plot the results in Figure 1 for selected values of $R \equiv c_{g} / \sigma_{s, x}$. Thick lines are for thick disks with scale heights $H_{g}=170 \mathrm{pc}$ and $H_{s}=330 \mathrm{pc}$. Results from Rafikov (2001) for razor-thin disks are also plotted as thin lines for comparison. The parameter domain below each marginal line corresponds to the stable regime. It is apparent that axisymmetric gravitational instability is mainly determined by the gaseous component for small $R$ and $Q_{s} \gtrsim 1$. When $R$ is small, the characteristic unstable length scales in (stand-alone) gaseous disks are much smaller than those of the stellar disk. In this case, the relative dominance of the gaseous to stellar components changes rather abruptly as $Q_{g}$ and $Q_{s}$ vary along a given marginal stability line, as manifested by the cusp in the $R=0.1$ line. As $R$ increases, the distinction between the characteristic length scales in the gas and stars becomes smaller, and the marginal line bends smoothly. A system with solar neighborhood parameters, marked by the filled circle at $\left(Q_{s}, Q_{g}\right)=(2.1,1.4)$, is highly stable to axisymmetric modes when treated as thick, while it would be marginally stable under the thin-disk approximation.

For varying disk thicknesses, Figure 2 plots the $Q_{g \text {,crit }}$ values and the corresponding marginal wavelength of perturbations, $\lambda_{\text {marg }}\left(\lambda<\lambda_{\text {marg }}\right.$ modes are stable). Self-gravity weakens when $H_{g}, H_{s}$, or $Q_{s}$ increases, tending to require lower $Q_{g \text {,crit }}$. A razorthin gas-only disk has $Q_{g \text {,crit }}=1.0$. This value increases to $Q_{g, \text { crit }}=1.27$ if the contribution from a thin stellar component with $Q_{s}=2.1$ is considered. For disks with thicknesses $H_{g}=0.87 c_{g} / \kappa$ and $H_{s}=0.4 \sigma_{s, x} / \kappa$ with $Q_{s}=2.1$ (similar to the solar neighborhood conditions), however, we have $Q_{g \text {, crit }}=0.67$ at $\lambda_{\text {marg }} \sim$ $2.3 \mathrm{kpc}$, as marked by filled circles in Figure 2 . This indicates that the stabilization caused by finite disk thickness is considerable, even larger than the destabilizing effect of stars. Solutions of equation (8) show that when $H_{g}=0.87 c_{g} / \kappa$ and $H_{s}=0.4 \sigma_{s, x} / \kappa$ are fixed, thick disks with $Q_{g}>1.4$ are stable to axisymmetric perturbations unless $Q_{s}<0.8$ (which is a very unrealistic range). Therefore, the naive expectation that including a stellar component

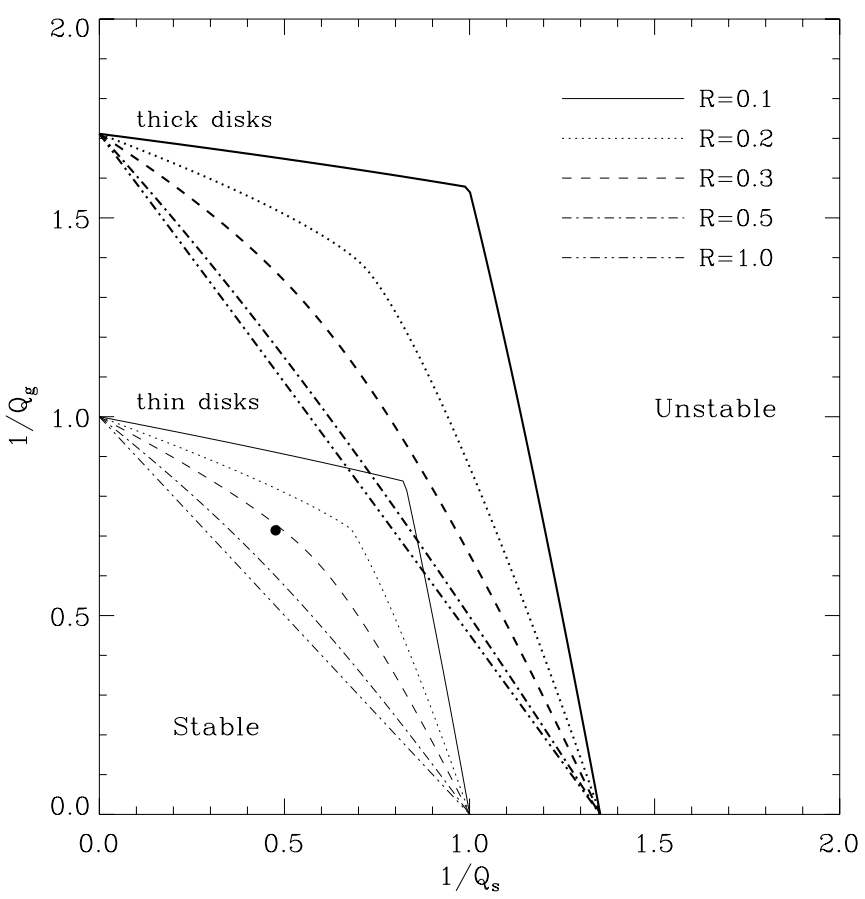

FIG. 1.-Marginal stability lines in the $Q_{s}-Q_{g}$ plane for axisymmetric gravitational instability in two-component disks with $R \equiv c_{g} / \sigma_{x}$. The thick lines correspond to thick disks with $H_{g}=170 \mathrm{pc}$ and $H_{s}=330 \mathrm{pc}$, while the thin lines are for razor-thin disks. The regions below each marginal line represent conditions for which two-component disks are axisymmetrically stable. The filled circle at $\left(Q_{s}, Q_{g}\right)=(2.1,1.4)$ marks solar neighborhood parameter values which are very stable when the disk is treated as thick, but would be barely stable if the disk were assumed razor thin.

increases $Q_{g, \text { crit }}$ over unity fails in disks with realistic thickness. The fact that $Q_{g \text {,th }} \sim 1.4$ for observed star formation thresholds in disk galaxies has often been attributed to gravitational instabilities in two-component systems (e.g., Kennicutt 1997). However, our results show that axisymmetric modes cannot be providing the needed instability when disk thickness is properly considered. We will show in $\S 5$ that instead, swing amplification of nonaxisymmetric perturbations are in fact able to drive two-component thick disks with $Q_{g} \lesssim 1.4$ into eventual gravitational runaway.

\section{NUMERICAL METHOD AND CODE TESTS}

\subsection{Numerical Method}

We have run a number of two-dimensional simulations for star plus gas systems, varying $Q_{g}$ and the number of stellar particles while fixing $H_{g}, H_{s}$, and $Q_{s}$. All the models have $256 \times 256$ resolution. We follow the nonlinear evolution of the gaseous part using the same version of the ZEUS code (Stone \& Norman 1992) as in Paper I; the reader is referred to Paper I for a detailed description of the code and the boundary conditions we adopt. In this subsection we describe the numerical methods mainly for the stellar part.

We use up to $N_{\mathrm{ptl}}=2 \times 10^{6}$ particles to represent a stellar disk and evolve them based on a particle-mesh (PM) algorithm. With its high velocity dispersion, the stellar component has a characteristic Jeans scale much larger than that of gas, so the resolution limit imposed by the PM method is entirely tolerable. In addition, the relaxation time of the particles amounts to $t_{R}=\sigma_{s, x}^{3} \Delta x /$ $\left(\pi G^{2} \Sigma_{s 0} m\right) \sim 1 \times 10^{3} t_{\text {orb }}$ for the parameters we adopt, where $\Delta x$ is the grid size and $m$ is the mass of individual particles (Rybicki 1971). This time is long enough to ensure that the evolution of the 

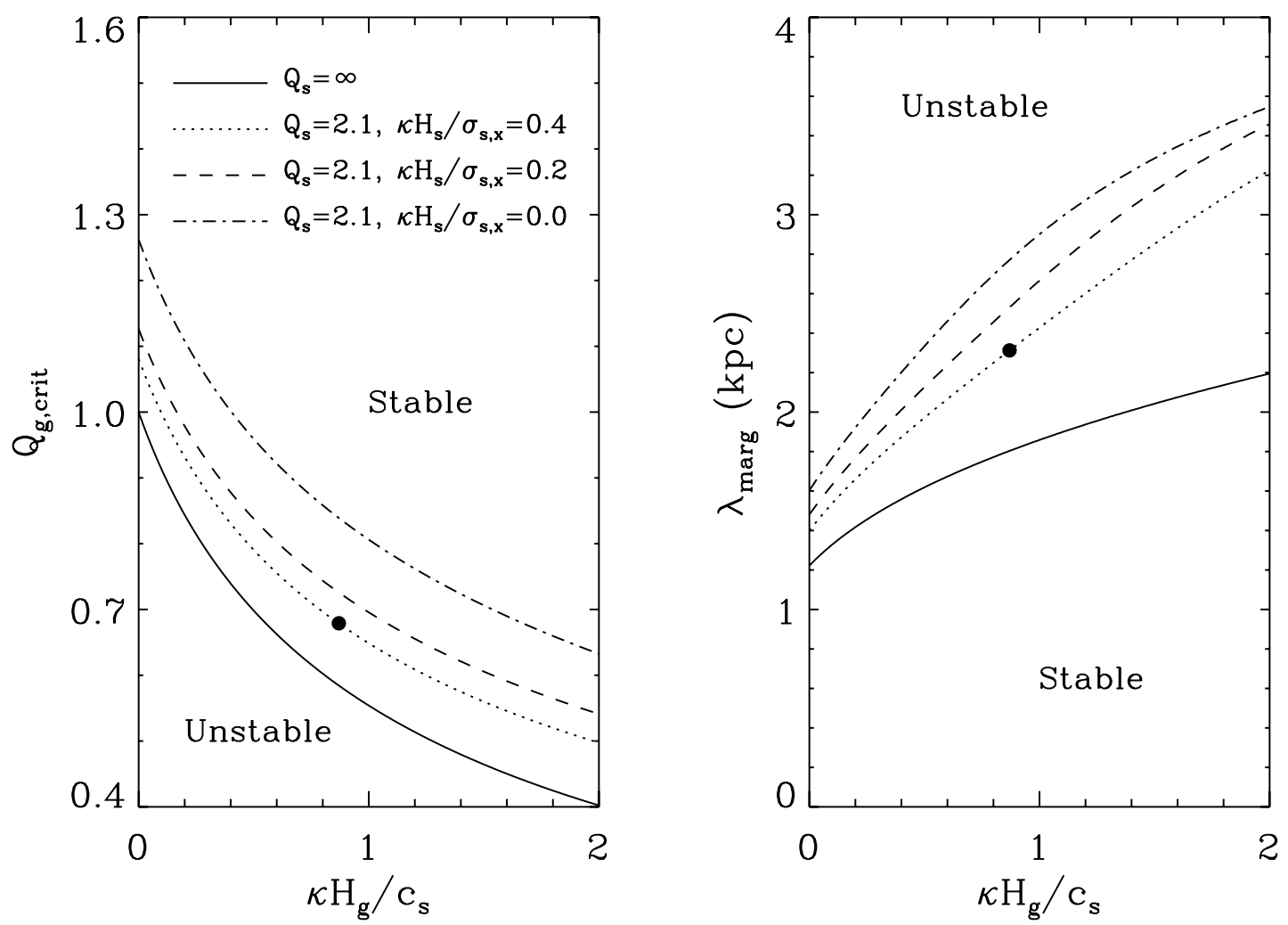

FIG. 2.-Critical $Q_{g}$ values (left) and the marginal perturbation wavelength $\lambda_{\text {marg }}(r i g h t)$ as functions of the gaseous scale height $H_{g}$. Regions with $Q_{g}<Q_{g \text {,rit }}$ or $\lambda>\lambda_{\text {marg }}$ correspond to an unstable parameter set. The solid lines correspond to the gas-only systems, while other lines are for combined disks with $Q_{s}=2.1$. The filled circles in both panels represent the solution for solar neighborhood parameters.

stellar system in our PM code is not seriously influenced by particle noise and relaxation (e.g., White 1988).

The initial distribution (eq. [5]) of the particle positions and velocities are realized using a pseudorandom number generator. At each time step, the stellar surface density $\Sigma_{s}$ is calculated on a $256 \times 256$ mesh via the triangular-shaped-cloud assignment scheme (Hockney \& Eastwood 1988). We solve equation (4) by employing the fast Fourier transform method on a sheared coordinate grid in which the surface density is perfectly periodic (Gammie 2001). The calculated potential is differentiated to give the gravitational force field at Cartesian mesh zone centers, which is then interpolated back to the particle locations. We integrate equation (1) using a modified predictor-corrector scheme that is second order accurate in time (e.g., Monaghan 1989). To handle the kinematics of the background shear self-consistently, we apply the shearing box boundary conditions in which $y$-boundaries are perfectly periodic and the $x$-boundaries are shearing periodic (Hawley et al. 1995; Huber \& Pfenniger 2001). These boundary conditions are fully consistent with our local models; particles leaving the simulation box from one $x$-face reenter with shifted $y$-positions through the opposite $x$-face.

\subsection{Code Tests}

We have checked our numerical methods on a number of test problems: one-dimensional or two-dimensional waves in gaseous or stellar disks separately or in combination. In this subsection we present the results of three tests that not only verify the accuracy of our numerical code, but also provide information on the necessary number of stellar particles to prevent numerical artifacts in the evolution of a self-gravitating stellar system.

Figure 3 plots the test results of axisymmetric density waves in razor-thin stellar disks. For these tests we consider one-dimensional,

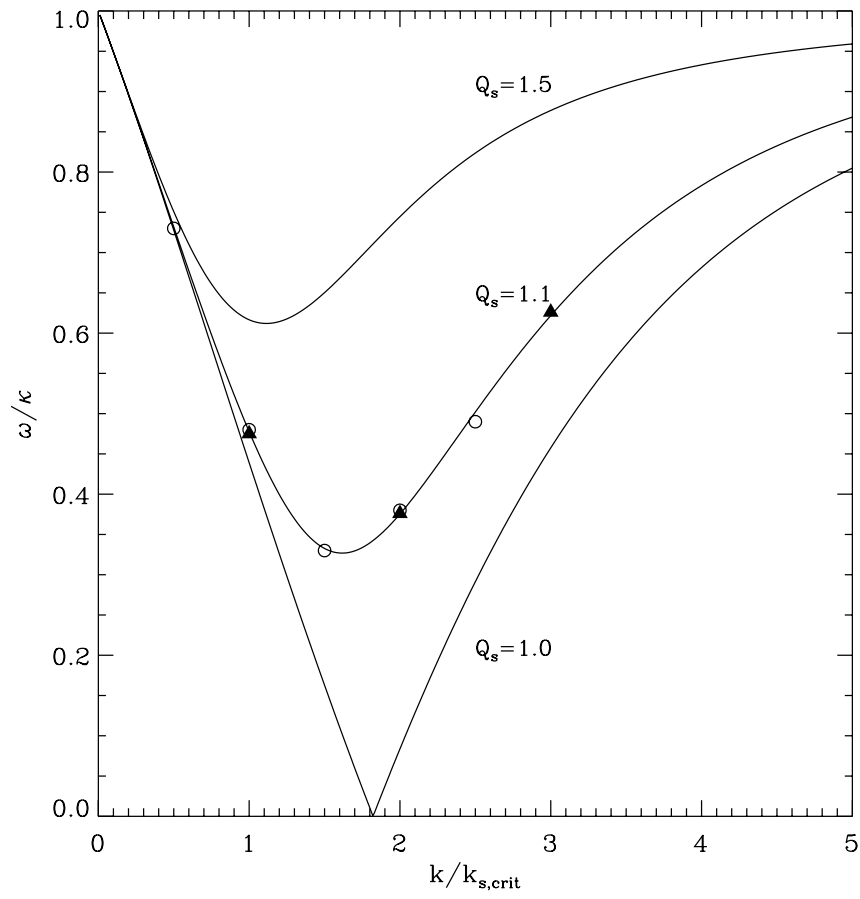

FIG. 3.-Oscillation frequencies of axisymmetric stellar density waves. The abscissa is the wavenumber $k$ normalized by $k_{s, \text { crit }} \equiv \kappa^{2} /\left(2 \pi G \Sigma_{s}\right)$. One-dimensional, razor-thin, star-only disks with $Q_{s}=1.1$ are adopted, and $N_{\mathrm{ptl}}=10^{4}$ particles are used. Open circles and filled triangles are from the $X=2$ and 1 models, respectively, both of which are in good agreement with the analytic solutions (lines) of eq. (8). 


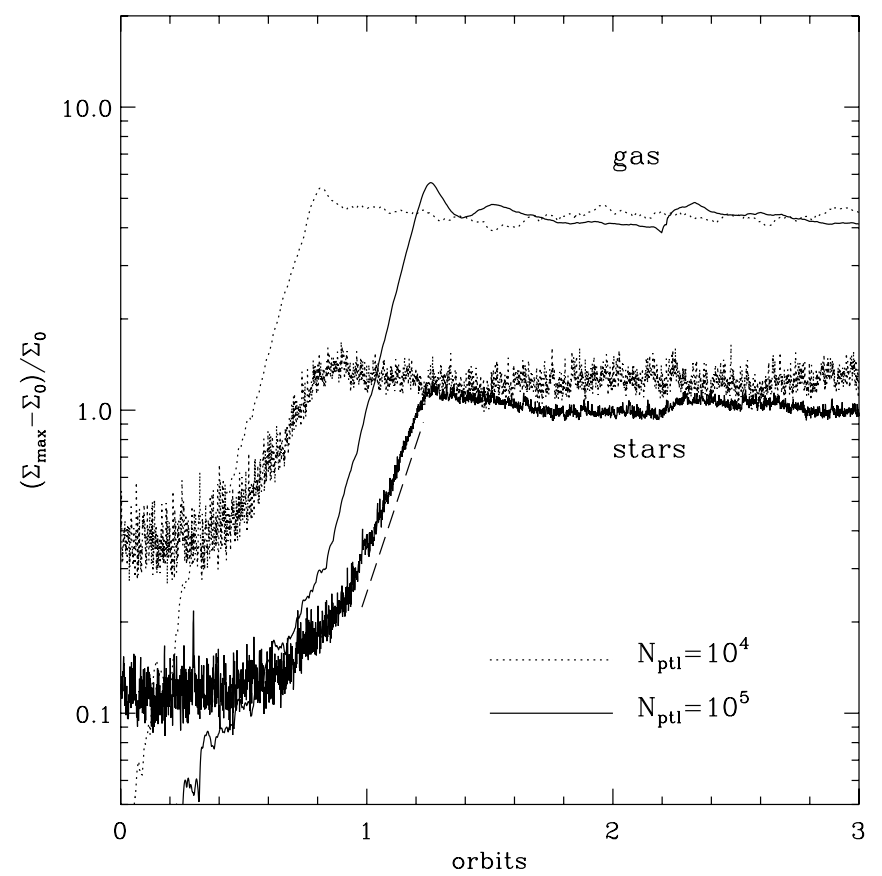

FIG. 4.- Time evolution of maximum surface densities of gas and stars in a combined disk with $q=1, Q_{g}=1.2, Q_{s}=1.5, H_{g}=0.05 c_{g} / \kappa, H_{s}=0.1 \sigma_{x} / \kappa$, and $c_{g} / \sigma_{x}=0.4$. An adiabatic equation of state with $\gamma=5 / 3$ is assumed for the gas. The long-dashed line gives the theoretical growth rate of $\sim 0.894 \Omega_{0}$, which is in good agreement with the results of numerical simulations.

star-only disks with $H_{s}=0, q=1, Q_{s}=1.1$ (close to the marginal state), and $X=1$ or 2 . For each run, $N_{\text {ptl }}=10^{4}$ particles are used. Frequencies obtained from the spatial and temporal Fourier analyses of the surface densities are given as filled triangles and open circles for the $X=1$ and 2 cases, respectively. The numerical results are within $\sim 2.5 \%$ of the analytic predictions (solid lines) from equation (8) for the chosen set of disk parameters. This confirms the stellar code's performance.

The second test addresses axisymmetric gravitational instability of a two-component disk, for which we choose $Q_{g}=1.2, Q_{s}=$ $1.5, q=1, H_{g}=0.05 c_{g} / \kappa, H_{s}=0.1 \sigma_{s, x} / \kappa$, and $c_{g} / \sigma_{s, x}=0.4$. The linear theory in $\S 3$ predicts that such a combined disk is unstable with a maximum growth rate of $\sim 0.894 \Omega_{0}$, which occurs at $\lambda_{\max }=3.53 \sigma_{s, x} / \kappa$. Setting the one-dimensional box size $L=\lambda_{\max }$ and assuming the gas is adiabatic with index $\gamma=5 / 3$, we have run two models with differing $N_{\text {ptl }}$. Figure 4 plots the resulting time histories of the maximum surface densities in both gaseous and stel- lar components. The dotted and solid lines correspond to $N_{\mathrm{ptl}}=$ $10^{4}$ and $10^{5}$ cases, respectively. Poisson noise in the stellar distribution immediately imposes (via gravity) strong density perturbations in the gaseous disk, which would otherwise be uniform. The perturbations in stars and gas soon become coherent and grow exponentially. The model with $N_{\mathrm{ptl}}=10^{4}$ has larger Poisson noise; hence, unstable modes grow sooner. Note that the growth rate of stellar surface density in both models is in good agreement with the theoretical estimate for the fastest growing mode, as represented by the long-dashed line in Figure 4. The gaseous component, already driven nonlinear, has slightly faster growth.

Finally, we report on the results of an important two-dimensional test. Evolution of linear waves in two-dimensional, shearing disks in principle would provide stringent tests for the current purposes, but we are unaware of test problems that have analytic solutions for comparison with numerical results. Swing amplification in a combined disk would also be a useful test, but it is difficult to accurately measure the amplification magnitude from numerical simulations. Propagating nonaxisymmetric waves in a shearing disk also would be an excellent test problem for the gas component (e.g., Paper I), but because of the Lagrangian character it does not work as well for a stellar disk. We thus consider evolution of nonaxisymmetric perturbations in nonshearing disks, for which equation (8) still holds true if $q=0$ and $k=\left(k_{x}^{2}+k_{y}^{2}\right)^{1 / 2}$ are used. We have run a set of numerical simulations for stable/unstable waves in various types of nonshearing disks and confirmed that numerical results are consistent with the analytic predictions, verifying again the accuracy of our numerical code.

Of particular interest is the effect of Poisson noise on gravitational instability in a stellar (or combined) system near the marginal state. Figure 5 plots the snapshots of surface density (in logarithmic scale) at $t / t_{\text {orb }}=7.0$ in a razor-thin, rigidly rotating, star-only disk with $q=0, Q_{s}=1.2$, and $X=2$. Although the linear theory suggests that a disk with these parameters should be stable, the maximum surface density in a model with $N_{\text {ptl }}=10^{4}$ grows secularly, prompting the formation of four clumps, as the left panel of Figure 5 shows. The growth of perturbations in a model with $N_{\text {ptl }}=10^{5}$ is slower, displaying overdensity honeycomb structures (Fig. 5, middle), while a model with $N_{\text {ptl }}=10^{6}$ has a stable density field oscillating mildly (Fig. 5, right). The mass assignment scheme in our two-dimensional PM code yields Poisson noise at the level $\delta \Sigma_{s 0} / \Sigma_{s 0}=1.44\left(N_{\mathrm{ptt}} / 10^{4}\right)^{-1 / 2}$, as measured by the standard deviation $\delta \Sigma_{s 0}$ of the initial surface density $\Sigma_{s 0}$. Some fraction of this noise may add to $\Sigma_{s 0}$, decreasing the effective value of $Q_{s}$. In cases when $N_{\text {ptl }}$ is small and when $Q_{s}$ is slightly larger than unity, $Q_{s, \text { eff }}$ can be smaller than the critical value and
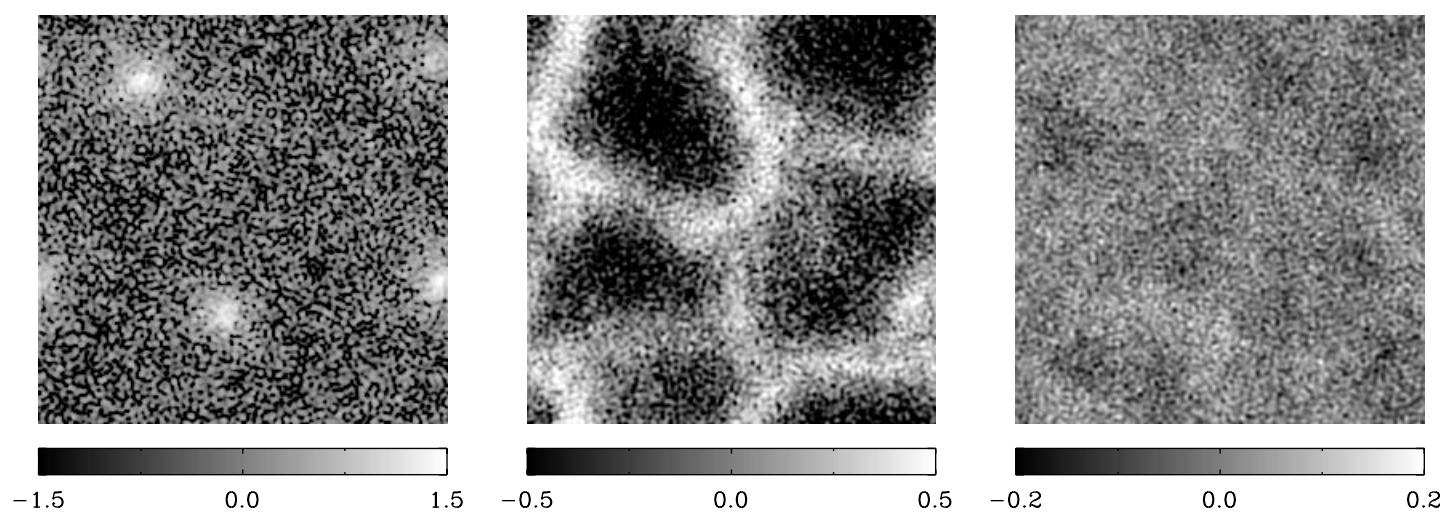

FIG. 5.- Snapshots of surface density at $t / t_{\text {orb }}=7.0$ in nonshearing, star-only systems with $Q_{s}=1.2, X=2, H_{s}=0$, but with differing particle numbers: $N_{\text {ptl }}=10^{4}$ (left); $N_{\mathrm{ptl}}=10^{5}$ (middle); and $N_{\mathrm{ptl}}=10^{6}\left(\right.$ right). Grayscale bars give $\log \left(\Sigma_{s} / \Sigma_{s 0}\right)$. It is apparent that large Poisson noise associated with small $N_{\mathrm{ptl}}$ effectively increases the surface density locally, lowering $Q_{s}$ and thus destabilizing systems that would otherwise remain stable. 

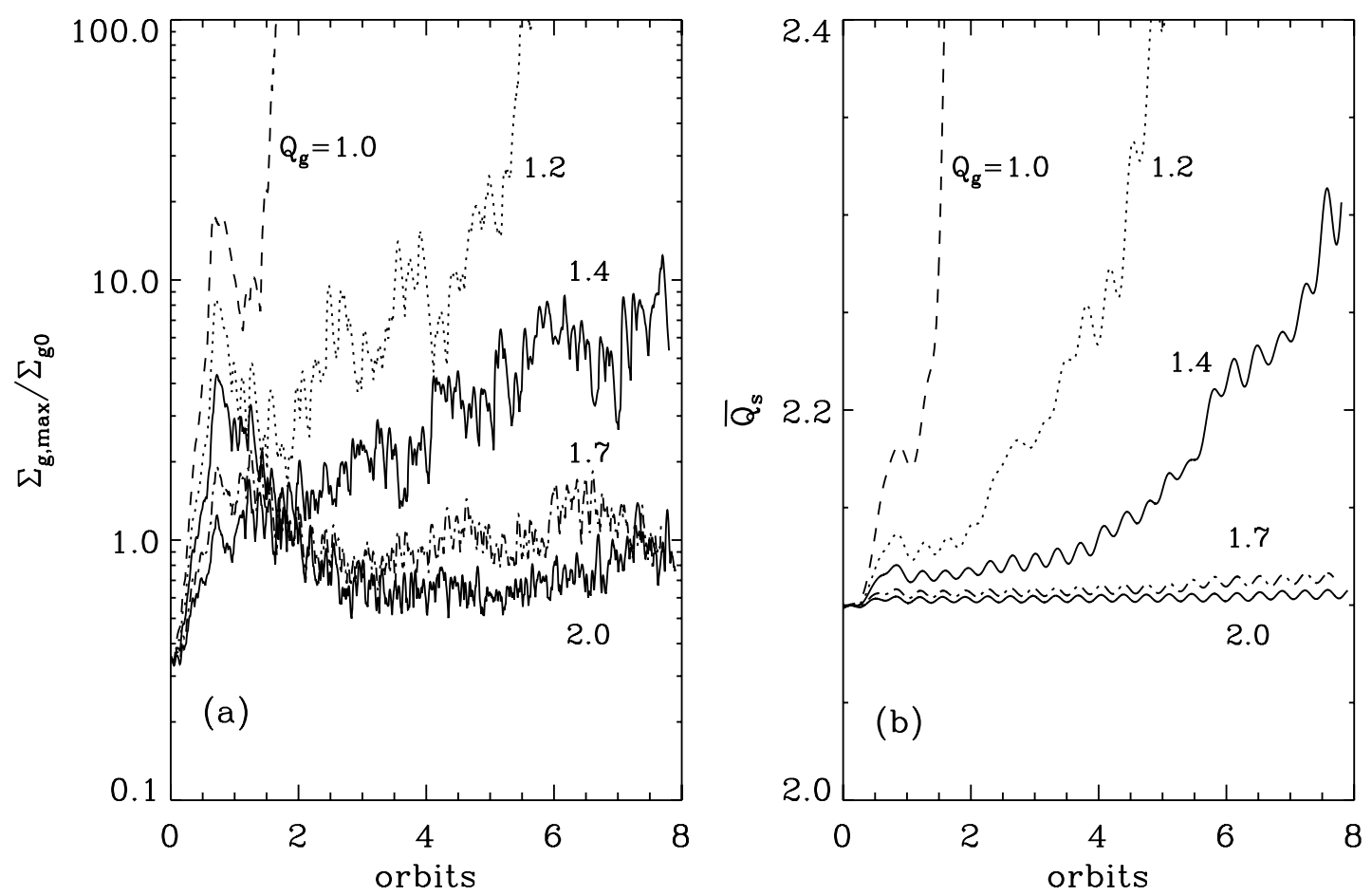

FIG. 6.-Evolution of the maximum gaseous surface density (left) and the spatially averaged stellar Toomre parameter $\bar{Q}_{s}$ (right), for models with various $Q_{g}$. All the models are two-dimensional and employ $2 \times 10^{6}$ stellar particles with $Q_{s, \text { init }}=2.1$. Models with $Q_{g} \leq 1.2$ experience gravitational runaway, while models with $Q_{g} \geq 1.7$ are stable; the $Q_{g}=1.4$ model is marginal with a rapidly fluctuating density field. For unstable or marginal models, $\bar{Q}_{s}$ increases considerably over time due to the gravitational interactions of stellar particles with both gaseous and stellar concentrations.

perturbations may grow artificially. ${ }^{4}$ This implies that Poisson noise can erroneously alter the fate of a numerically modeled stellar system, especially when it is close to being marginal.

This point has sometimes been overlooked in previous $N$-body simulations of disks. For example, Griv et al. (1999) simulated dynamics of nonshearing, local stellar disks using about 6500 particles and found that disks with $1<Q_{s 0} \lesssim 1.5$ were unstable. They argued that the apparent discrepancy between the numerical and theoretical results might be partly because the WKB theory of Lin $\&$ Shu (1966) for density waves is incomplete and partly because their method for computing self-gravity is not very accurate. We suggest that large Poisson noise alone, simply associated with a small number of particles, can explain their numerical results. Adequate resolution is particularly an issue for global disk models, since the Poisson noise on a given spatial scale is proportional to the linear size of the disk. Some of the global simulations of $\mathrm{Li}$ et al. $\left(2005,2006\right.$; with $0.2-1.2 \times 10^{6}$ star particles for the whole disk), for example, may be affected by amplification of noise.

\section{NONLINEAR SIMULATIONS}

To investigate nonlinear evolution of two-component differentially rotating disks with a range of gas mass fractions, we have run a number of nonaxisymmetric models. We fix $q=1, Q_{s}=2.1$, $X=2, \kappa H_{s} / \sigma_{s, x}=0.4, \kappa H_{g} / c_{g}=0.87$, and vary $Q_{g}$ in the range of 1-3. For these parameters, axisymmetric perturbations are stable. Solutions for self-consistent vertical equilibria show that $H_{g}$ decreases only by $\sim 20 \%$ as $Q_{g}$ decreases from 1.4 to 1.0 (Kim et al. 2002), so that we expect using a fixed value of $H_{g}$ will not significantly impact our quantitative results.

\footnotetext{
${ }^{4}$ If a half of $\delta \Sigma_{s 0}$ contributes directly to $\Sigma_{s 0}$, the effective Toomre parameter becomes $Q_{s, \text { eff }}=Q_{s}\left(1+\delta \Sigma_{s 0} / 2 \Sigma_{s 0}\right)^{-1}$. When $Q_{s}=1.2, Q_{s, \text { eff }}=0.70,0.98$, and 1.12 for $N_{\mathrm{ptl}}=10^{4}, 10^{5}$, and $10^{6}$, respectively, which is roughly consistent with the results of our numerical experiments.
}

We set up initially uniform gaseous and stellar disks and apply density perturbations only to the gaseous component. Because of randomness in particle locations, the stellar part already has whitenoise density perturbations with amplitudes of $\sim 10 \%$ when $N_{\mathrm{ptl}}=$ $2 \times 10^{6}$ which will be immediately transmitted into the gaseous disk. In order to keep the Poisson noise from dominating the evolution of the gaseous disk, we impose rather strong gaseous density perturbations using a Gaussian random field with a power spectrum $\left\langle\left|\Sigma_{g, k}^{2}\right|\right\rangle \propto k^{-8 / 3}$ for $1 \leq k L /(2 \pi) \leq 256$ in Fourier space. This corresponds to a two-dimensional Kolmogorov spectrum if wave motions obey a sonic dispersion relation (Paper I). We measure the standard deviation of the gas surface density in real space and fix it to $10 \%$. Although this level of density fluctuations is chosen as a compromise between maintaining the initial perturbations in the linear regime and reducing the effect of Poisson noise on the simulation outcomes, it may in fact well represent initial conditions for the highly turbulent ISM observed in real disk galaxies.

\subsection{Threshold for Gravitational Runaway}

We begin with a high-resolution model with $Q_{g}=1.0$ and $N_{\mathrm{ptl}}=$ $2 \times 10^{6}$. For this model, the combined gravity of gas and stars induces efficient swing amplification, leading to gravitational runaway. Because disk thickness dilutes self-gravity, each disk (i.e., purely gaseous or purely stellar) in isolation would have remained stable both to axisymmetric and nonaxisymmetric perturbations. Figure 6 plots as dashed lines the evolution of the maximum gaseous surface density and the spatially averaged, stellar Toomre parameter $\bar{Q}_{s}$ of this model (together with those of other models). Figure 7 displays snapshots of gas and stellar surface density in this model at three time epochs, while Figure 8 shows the initial modal growth of perturbations in the gas surface density over time. 

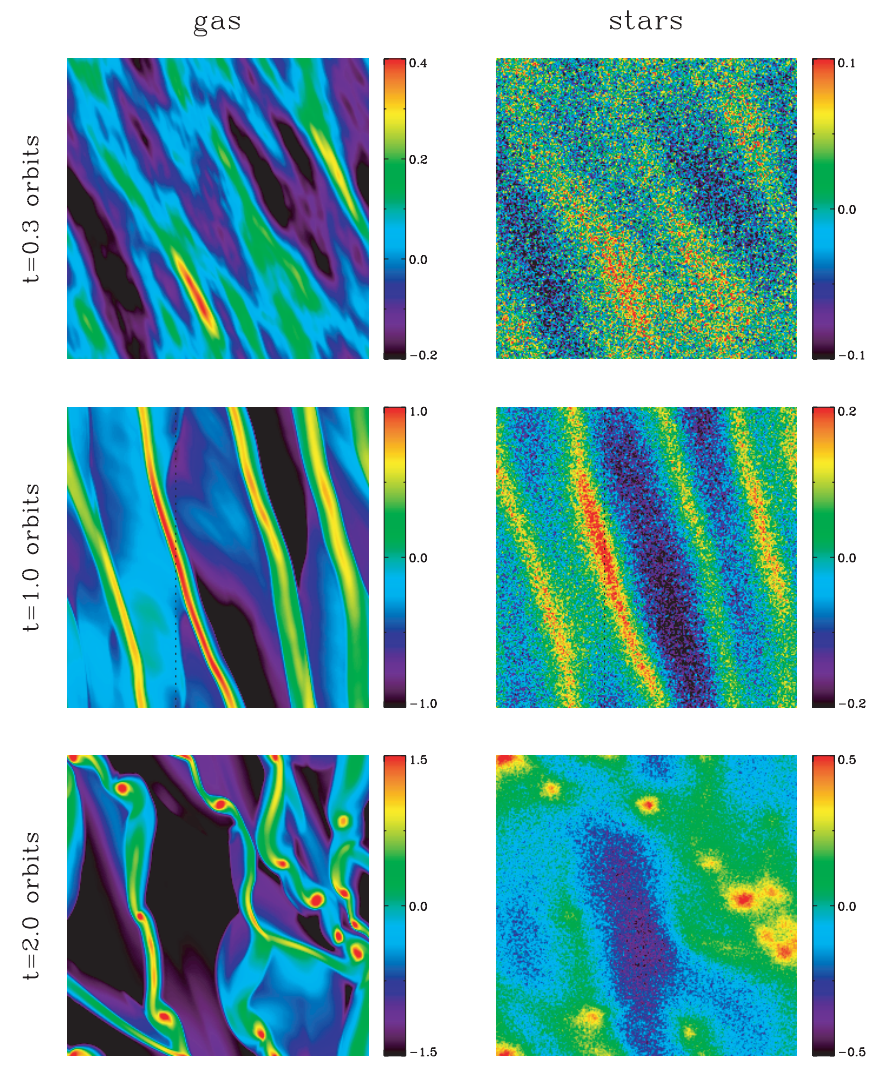

FIG. 7.-Snapshots of gaseous (left) and stellar density (right) in logarithmic color scale for the $Q_{g}=1.0$ model. Dotted lines in black in the middle row indicate the $x$-position at which slices of various physical quantities are taken in Fig. 9. Strong filaments develop in the gas, and weaker filaments in the stars, due to swing amplification ( $t=1.0$ orbits). The gaseous filaments subsequently gravitationally fragment into clumps ( $t=2.0$ orbits).

Initially, density perturbations (a superposition of modes with different wavenumbers) adjust by launching sound waves in the gas and redistributing stellar particles on their epicyclic orbits. During this relaxation phase, which lasts $\sim 0.2 t_{\mathrm{orb}}$, coherent perturbations in stars and gas begin to develop through gravitational interactions. Because of the uniform background shear, the radial wavenumber $k_{x}(t)$ of perturbations increases linearly with time as

$$
k_{x}(t)=k_{x}(0)+q \Omega_{0} k_{y} t,
$$

where $k_{y}$ denotes the $y$-wavenumber and $k_{x}(0)$ is the initial $x$ wavenumber of perturbations. This kinematic shift of $k_{x}(t)$ causes wave fronts to swing from leading to trailing. Epicyclic motion of both stellar particles and gaseous fluid elements has the same rotational sense as the wave fronts, extending their exposure to selfgravity as they linger in wave crests. Perturbations keep growing until the radial wavenumber becomes large, forming overdense, trailing wavelets (Goldreich \& Lynden-Bell 1965b; Julian \& Toomre 1966; Toomre 1981; Paper I).

The modal growth of density perturbations via swing amplification in the $Q_{g}=1.0$ model is well illustrated in Figure 8 where we plot the evolution of power spectra of the gaseous surface density as functions of the normalized wavenumber $n_{x}(t) \equiv L k_{x}(t) /$ $(2 \pi)$. Only the modes with $k_{y}=2 \pi / L$ and $\left|n_{x}(0)\right| \leq 3$ that have large amplitudes are shown. Note that a unity increment of $n_{x}(t)$ along each line corresponds to a time elapse of $\Delta t=(2 \pi)^{-1} t_{\mathrm{orb}}$. It is apparent that swing amplification is active only when waves are loosely wound with $\left|n_{x}(t)\right| \lesssim 3-4$, above which sonic oscil-

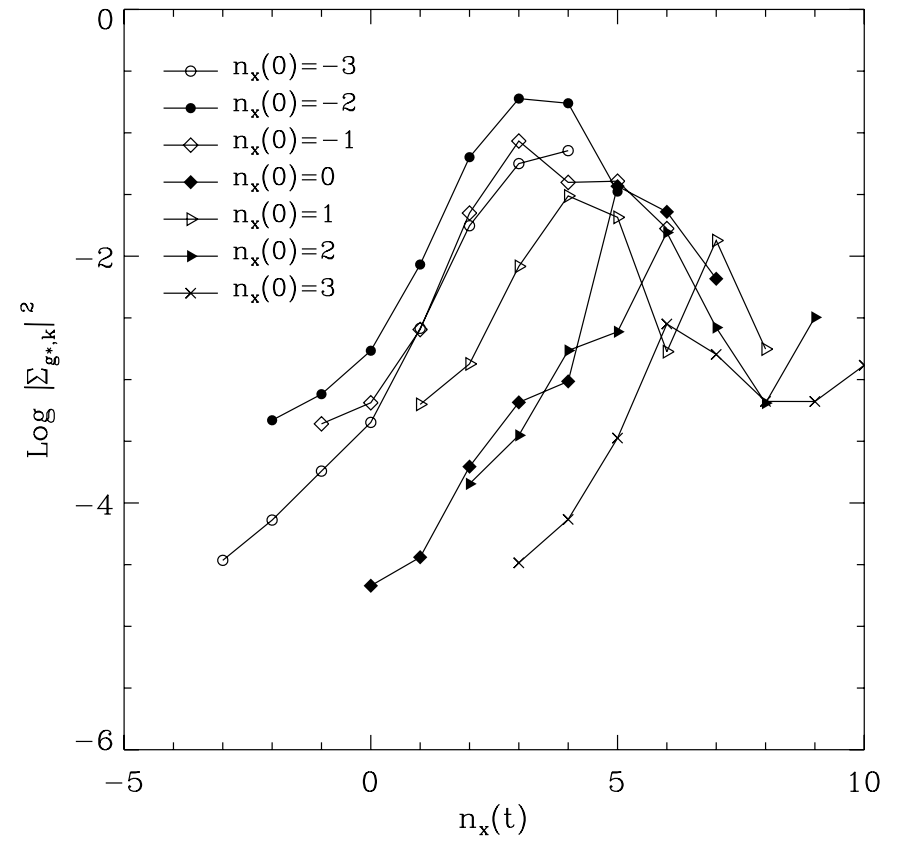

FIG. 8. - Modal growth of power spectra $\left|\Sigma_{g, k}\right|^{2}$ of the gaseous surface density against the normalized wavenumber $n_{x}(t) \equiv L k_{x}(t) /(2 \pi)$ in the $Q_{g}=1.0$ model for $t / t_{\text {orb }} \lesssim 1.1$. A few selected modes with $k_{y}=2 \pi / L$ and $\left|n_{x}(0)\right| \leq 3$ are shown. These loosely wound waves exhibit growth via swing amplification, then saturation. Note that $n_{x}(t)$ increases linearly with time, with $\Delta t=\Delta n_{x}(t) t_{\mathrm{orb}} /$ $(2 \pi)$. The $t=0$ point is the farthest left on each line.

lations quench the perturbation growth. The $n_{x}(0)=1$ mode that happens to have the largest initial power grows and emerges first, as manifested by the trailing modes with $n_{x}\left(t / t_{\text {orb }}=0.3\right) \approx 3$ shown in the top row of Figure 7. However, it is the modes with $n_{x}(0)<0$ that eventually dominate the initial swing amplification, since they have longer time for growth.

As Figures 6 and 8 show, the initial swing amplification in the $Q_{g}=1$ model saturates at around $t / t_{\text {orb }} \simeq 0.7$. At this time, the gaseous surface density reaches $\Sigma_{g \text {,max }} / \Sigma_{g 0}=18.7$, while the stellar component reaches only $\Sigma_{s, \max } / \Sigma_{s 0}=2.5$. The stellar component's density increases less due to its larger initial values of $Q_{s}$ and $H_{s}$ and the steady heating associated with particle scattering off shearing wavelets. While the maximum gas surface density is rather high when the swing amplifier saturates, the gas filaments are very thin. Reduction of self-gravity due to finite disk thickness prevents these filaments from immediately undergoing gravitational collapse. The filaments expand slightly, reducing their surface density. Some filaments have sufficient perturbed velocities to offset the tendency for wave rotation of the background shear. Consequently, the pitch angles of these high-density filaments vary little; they can be viewed as transient (swing-generated) spiral density waves in a two-component disk.

Figure 9 plots sample cut profiles of various quantities along the $x / L=-0.14$ dotted lines in the snapshot shown in the middle row of Figure 7. The gas filament has a pitch angle $i=16^{\circ}$ and is almost corotating with the center of the box; it coincides with a local stellar enhancement such that the composite may be considered a transient, local spiral arm segment. As the gas at $-0.18<$ $x / L<-0.2$ enters the arm, it is decelerated and compressed, analogous to a supersonic de Laval flow. This in turn increases the velocity parallel to the arm due to the constraint of potential vorticity conservation (e.g., Hunter 1964; Balbus \& Cowie 1985; Gammie 1996; Kim et al. 2002). Note that the gravitational potential is dominated by the gaseous component, which tends to symmetrize the density profile with respect to the peak (Lubow 

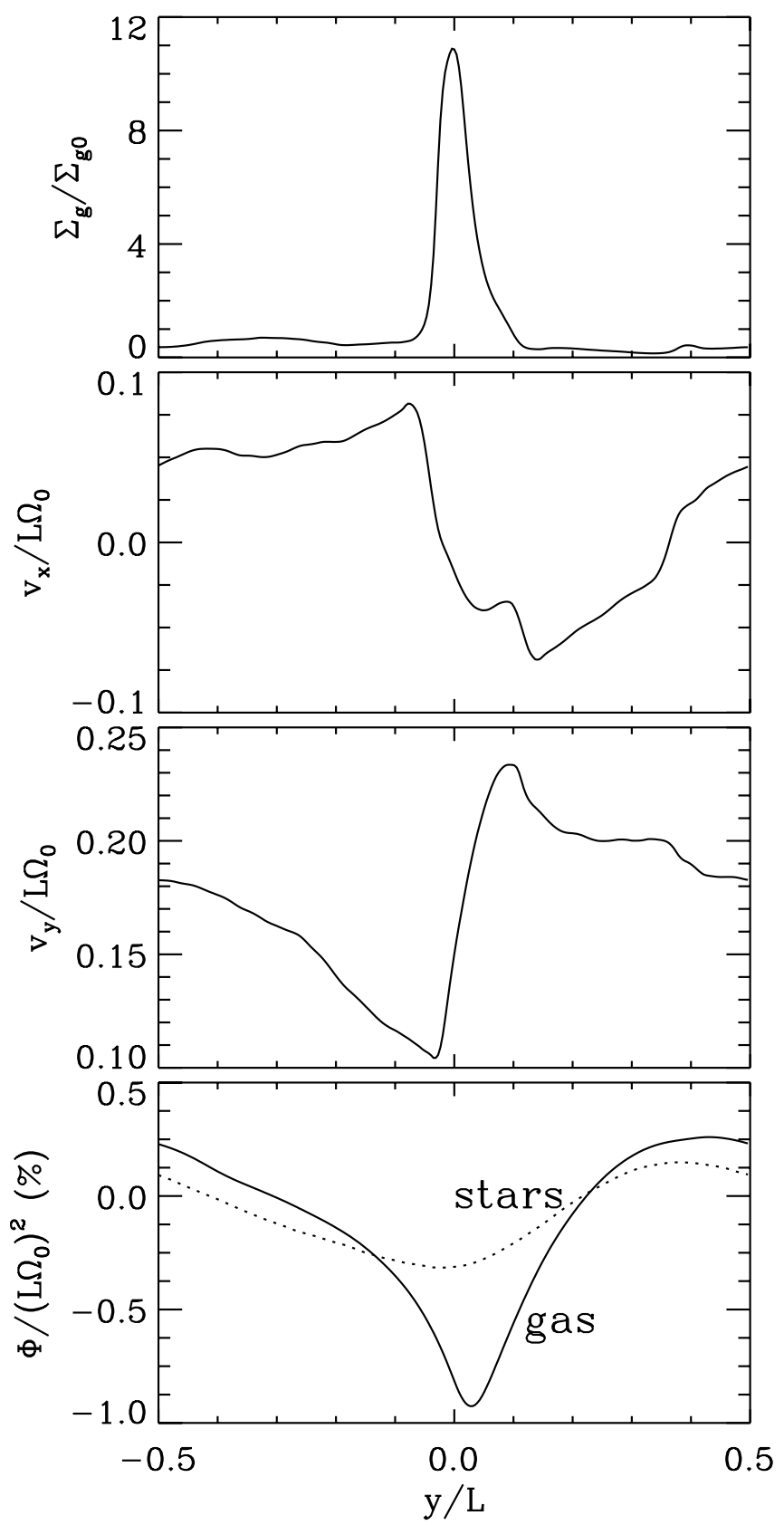

FIG. 9.- Profiles of gaseous surface density, gaseous velocities, and gravitational potentials across a filament at $x / L=-0.14$ when $t / t_{\text {orb }}=1$, for the $Q_{g}=$ 1.0 model. The profiles are qualitatively similar to those in galactic spiral shocks when both gas and stars are present (e.g., Lubow et al. 1986).

et al. 1986). The structure of this filament persists until $t / t_{\text {orb }} \sim 1.2$ when collisions with other filaments make it highly self-gravitating.

Paper I found that formation of bound clouds in swing-amplified disks occurs generically through one of the three secondary instabilities. In order of decreasing self-gravity, these are (1) parallel fragmentation of filaments, (2) gravitational collisions of shearing wavelets, and (3) rejuvenated swing amplification preceded by nonlinear wave interactions. As explained above, high-density filaments in the $Q_{g}=1.0$ model do not immediately fragment due to thick-disk gravity. Only after several collisions with neighboring filaments is the density high enough to form gravitationally bound condensations. As Figure 7 shows, the $Q_{g}=1.0$ model forms 14 bound gaseous clouds, the average mass of which is $\sim 6 \times$ $10^{7} M_{\odot}$, roughly 10 times larger than the Jeans mass $M_{\mathrm{J}, 2 \mathrm{D}}=$ $c_{g}^{4} /\left(G^{2} \Sigma_{g 0}\right)$ in the corresponding gas-only, razor-thin disk. For comparison, in the $Q_{g}=1$ models of Paper I, the condensations that formed had $M / M_{\mathrm{J}, 2 \mathrm{D}} \sim 0.5-1$, suggesting that the effect of finite disk thickness (combined with an active stellar disk) is nonnegligible in setting the cloud masses that form.

We note that the bound condensations that form in our models may represent $\mathrm{H}$ I superclouds observed in many disk galaxies (e.g., Elmegreen \& Elmegreen 1983; Knapen et al. 1993). In our models (isothermal, without feedback), these superclouds keep collapsing in a runaway fashion to the resolution limit. An artificial consequence of the runaway cloud collapse, at highly nonlinear stages, is the formation of loose stellar aggregates, as Figure 7 displays. Note that all the stellar clumps coincide with the massive gaseous clumps. In realistic situations, however, gaseous superclouds would presumably fragment into lower mass GMCs when appropriate physical processes such as internal turbulence are included. If $M_{\mathrm{sc}}$ and $R_{\mathrm{sc}}$ denote the mass and size of superclouds, respectively, stars would be drawn in strongly only if $R_{\mathrm{sc}}<G M_{\mathrm{sc}} / \sigma_{s, x}^{2} \sim 300 \mathrm{pc}$ for $M_{\mathrm{sc}}=6 \times 10^{7} M_{\odot}$ and $\sigma_{s, x}=$ $30 \mathrm{~km} \mathrm{~s}^{-1}$. This corresponds to a supercloud surface density $\Sigma_{\text {sc }}>200 M_{\odot} \mathrm{pc}^{-2}$, comparable to the typical surface density of a GMC. As long as GMCs are destroyed by star formation before the whole supercloud reaches a density as large as that of an individual GMC (which is observationally true), stellar clumps would not be produced in reality.

As shown in the right panel of Figure 6, the stellar velocity dispersion in this model continually increases as individual stars scatter off stellar and, more importantly, gaseous filaments formed via swing amplification. This process is somewhat analogous to heating of a stellar disk by transient spiral density waves (e.g., Barbanis \& Woltjer 1967; Sellwood \& Carlberg 1984; Carlberg \& Sellwood 1985; Fuchs 2001; De Simone et al. 2004; Minchev $\&$ Quillen 2006), although the runaway fragmentation and collapse of gaseous fragments in our two-component models cause the stellar velocity dispersions to rise more rapidly than in star-only systems.

Figure 6 shows that a model with $Q_{g}=1.2$ also becomes unstable, but with weaker gaseous gravity it takes longer to reach gravitational collapse. The first-generation filaments formed in this model do not fragment directly, and unlike in the $Q_{g}=1$ model, collisions do not yield merger-induced fragmentation. Instead, the structures nonlinearly interact with each other and supply fresh, small- $\left|k_{x}\right|$ modes that undergo subsequent swing amplification (Paper I; see also Fuchs et al. [2005] for its stellar analog). Four successive stages of "rejuvenated" swing amplification (from interactions of filaments of order unity amplitudes) are required to drive the $Q_{g}=1.2$ model into eventual gravitational runaway. The average mass of gaseous clouds that form is again $\sim 10 M_{\mathrm{J}, 2 \mathrm{D}}$.

When we further reduce the gas surface density so that $Q_{g}=$ 1.4 , the initial swing amplification yields a moderately selfgravitating state that never forms bound clumps over the course of the simulation. As Figure 10 shows, the gaseous component in this model is dominated by shearing wavelets (and the related velocity field). Mild rejuvenated swing amplification allows the peak gaseous surface density and velocities to grow steadily, reaching the nonlinear regime. The stellar velocity dispersions also grow, but the net increase is less than $10 \%$ at $t / t_{\text {orb }}=8$ (see Fig. 6 ); the stellar disk also remains virtually uniform with only very low fluctuations in density. We regard this model as "marginal" because it would almost certainly end up with bound condensations if evolved over a sufficiently long time. In other models with $Q_{g} \geq 1.7$, growth is so weak that perturbed surface densities remain in the linear regime at the saturation of the first swing amplification. Rejuvenation of swing is almost absent in these models, so that 

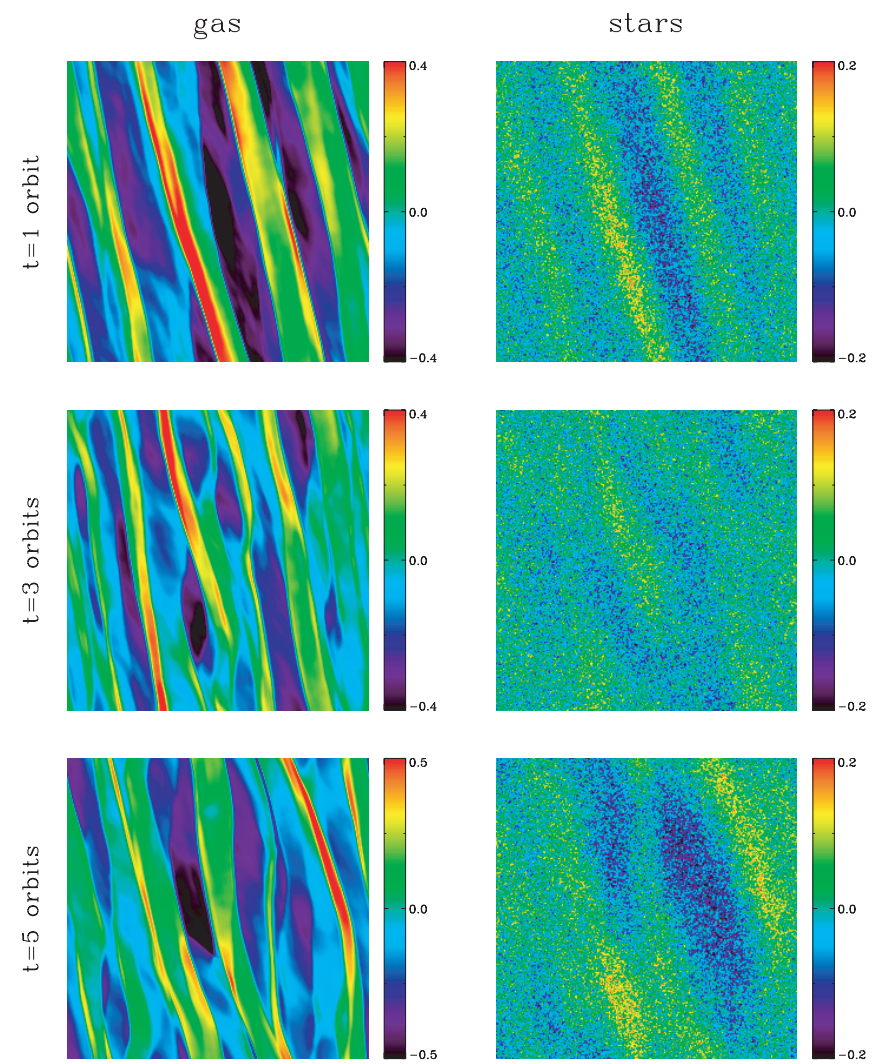

FIG. 10.-Snapshots of gaseous (left) and stellar density $(r i g h t)$ in logarithmic color scale for the $Q_{g}=1.4$ model. Note that nonlinear shearing wavelets are prevalent in the gaseous disk, while density fluctuations in the stellar disk are very weak.

the combined systems can be considered nonlinearly stable. We conclude, therefore, that the threshold for gravitational instability in combined gas-star disks has $Q_{g}$ near 1.4.

\subsection{Gravity-driven Turbulence}

We now examine the properties of turbulence generated by swing amplification in two-component disks. We define $\delta E_{\text {kin }} \equiv$ $\frac{1}{2} \int \Sigma_{g}\left(\boldsymbol{v}-\boldsymbol{v}_{0}\right)^{2} d x d y / \Sigma_{g 0}$, representing the specific kinetic energy associated with turbulent velocities in the gas. ${ }^{5}$ We have shown in $\S 4.2$ that large Poisson noise in a particle distribution effectively increases the mean surface density of stars in local regions and can even incur spurious gravitational instability. Similarly, random particle noise can artificially — and possibly significantly — enhance the saturated state value of $\delta E_{\text {kin }}$.

We want to measure $\delta E_{\text {kin }}$ in a way that minimizes the effect of particle noise. To this end we take the following steps. (1) Run a two-component model in which both stellar and gaseous disks evolve under the total (gaseous plus stellar) self-gravity and measure the resulting kinetic energy, which we denote by $\delta E_{\mathrm{kin}}^{\mathrm{SG}}$. (2) Run a control model with the same set of parameters as in step (1), but evolving the stellar component kinematically [i.e., omitting the $\nabla\left(\Phi_{s}+\Phi_{g}\right)$ gravity terms in eq. (1)], while evolving the gaseous component including both self- and stellar gravity. In runs of type (2), no initial perturbation is applied to the gas, but the gas responds to the Poisson noise of the stars. The energy resulting from runs of type (2) are denoted $\delta E_{\mathrm{kin}}^{\mathrm{NG}}$. (3) Calculate the gaseous velocity dispersion $\sigma_{v} \equiv\left(2\left\langle\delta E_{\mathrm{kin}}^{\mathrm{SG}}\right\rangle-2\left\langle\delta E_{\mathrm{kin}}^{\mathrm{NG}}\right\rangle\right)^{1 / 2}$, where the

\footnotetext{
5 Note that the total perturbed kinetic energy also contains other terms; $\delta E_{\text {kin }}$ is (half of) the mass-weighted mean-squared turbulent velocity dispersion.
}

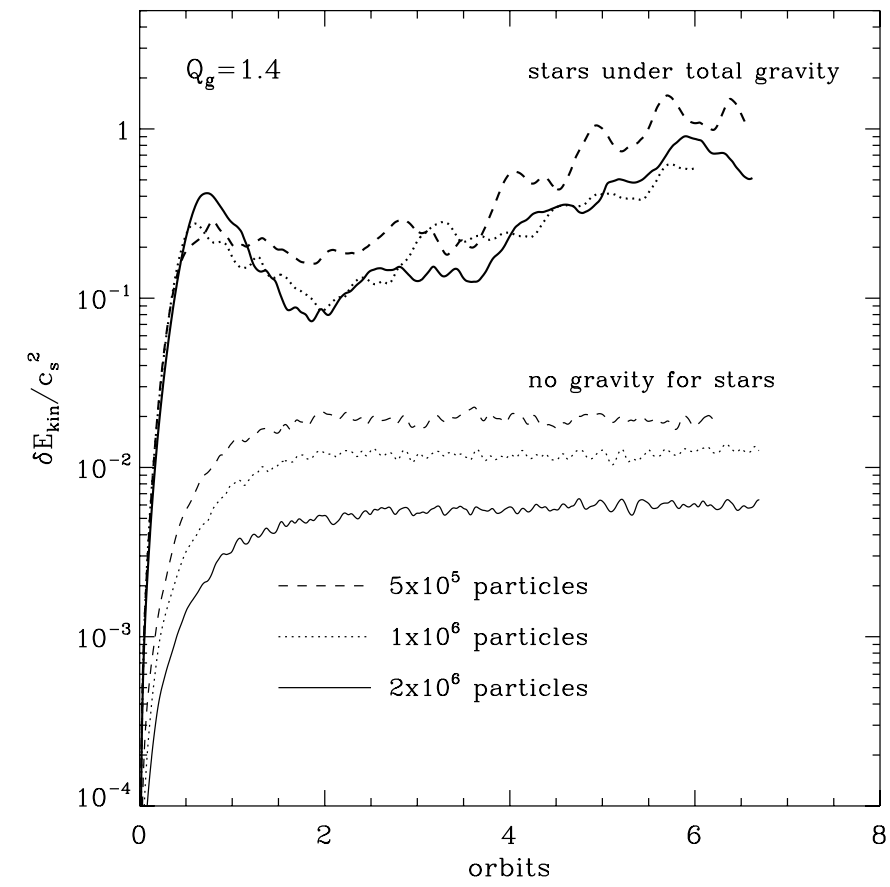

FIG. 11.-Evolutionary histories of the random kinetic energy of the gas, $\delta E_{\mathrm{kin}}=\frac{1}{2} \int \Sigma_{g}\left(\boldsymbol{v}-\boldsymbol{v}_{0}\right)^{2} d x d y / \Sigma_{g 0}$, in two-component disks with $Q_{g}=1.4$ and $Q_{s}=2.1$. Three cases with different $N_{\mathrm{ptl}}$ are shown. The thick lines are for the models where the both stellar and gaseous components are fully self-gravitating, while the thin lines correspond to the cases in which the combined gravity applies only to the gaseous disk. The stellar disk in the latter case evolves passively, but still imposes gravitational forcing from Poisson noise on the gas disk.

brackets $\langle\cdots\rangle$ denote a temporal average over some time interval (typically over $1-6$ orbits). (4) Finally, vary $N_{\text {ptl }}$ and repeat steps (1)-(3) to check the convergence of $\sigma_{v}$ with increasing $N_{\mathrm{ptt}}$.

Figure 11 displays evolutionary histories of $\delta E_{\text {kin }}$ for $Q_{q}=1.4$ models that differ in the number of particles and the treatment of gravity for the stellar component. The thick lines correspond to runs following (1) above, while thin lines correspond to runs following (2). In the cases with full gravity, the systems relax after the initial swing amplification, with $\delta E_{\mathrm{kin}}^{\mathrm{SG}}$ decreasing temporarily as some of the gaseous kinetic energy is transferred via sound waves and/or weak shocks into thermal energy that dissipates under the isothermal prescription. The continuous input from stellar perturbations as well as nonlinear feedback from the shearing wavelets cause a resumed increase in $\delta E_{\mathrm{kin}}^{\mathrm{SG}}$. After $t=2$ orbits, models with gravitating stars show a secular increase of $\delta E_{\text {kin }}^{\mathrm{SG}}$. On the other hand, in models where the stars evolve passively, the initial swing is weak enough that $\delta E_{\mathrm{kin}}^{\mathrm{NG}}$ simply saturates at a constant (low) value. Other models (not shown) with $Q_{g} \geq 2.0$ show that both $\delta E_{\mathrm{kin}}^{\mathrm{SG}}$ and $\delta E_{\mathrm{kin}}^{\mathrm{NG}}$ are more or less constant after $t / t_{\text {orb }}=2$, because swing amplification is very mild.

Figure $12 a$ plots the results for $\sigma_{v}$ against $N_{\mathrm{ptl}}$ as filled circles for various $Q_{g}$. In computing $\sigma_{v}$, we take time averages of $\delta E_{\mathrm{kin}}$ over $t / t_{\text {orb }}=1-6$ for all the models except the unstable model with $Q_{g}=1.2$. For $Q_{g}=1.2$, time averages are taken only over $t / t_{\text {orb }}=1-4$, since afterward the velocity field is mainly a response to discrete high-density filaments. For comparison, Figure $12 a$ also plots values of $\tilde{\sigma}_{v} \equiv\left\langle 2 \delta E_{\mathrm{kin}}^{\mathrm{SG}}\right\rangle^{1 / 2}$ as open circles for selected values of $Q_{g}$. The figure shows that for $Q_{g}<1.7, \sigma_{v}$ and $\tilde{\sigma}_{v}$ are in close agreement. More generally, it is clear that there are progressively smaller differences between $\sigma_{v}$ and $\tilde{\sigma}_{v}$ as $Q_{g}$ decreases, indicating that the contribution from swing-amplified Poisson noise to $\tilde{\sigma}_{v}$ becomes increasingly unimportant at lower $Q_{g}$. 

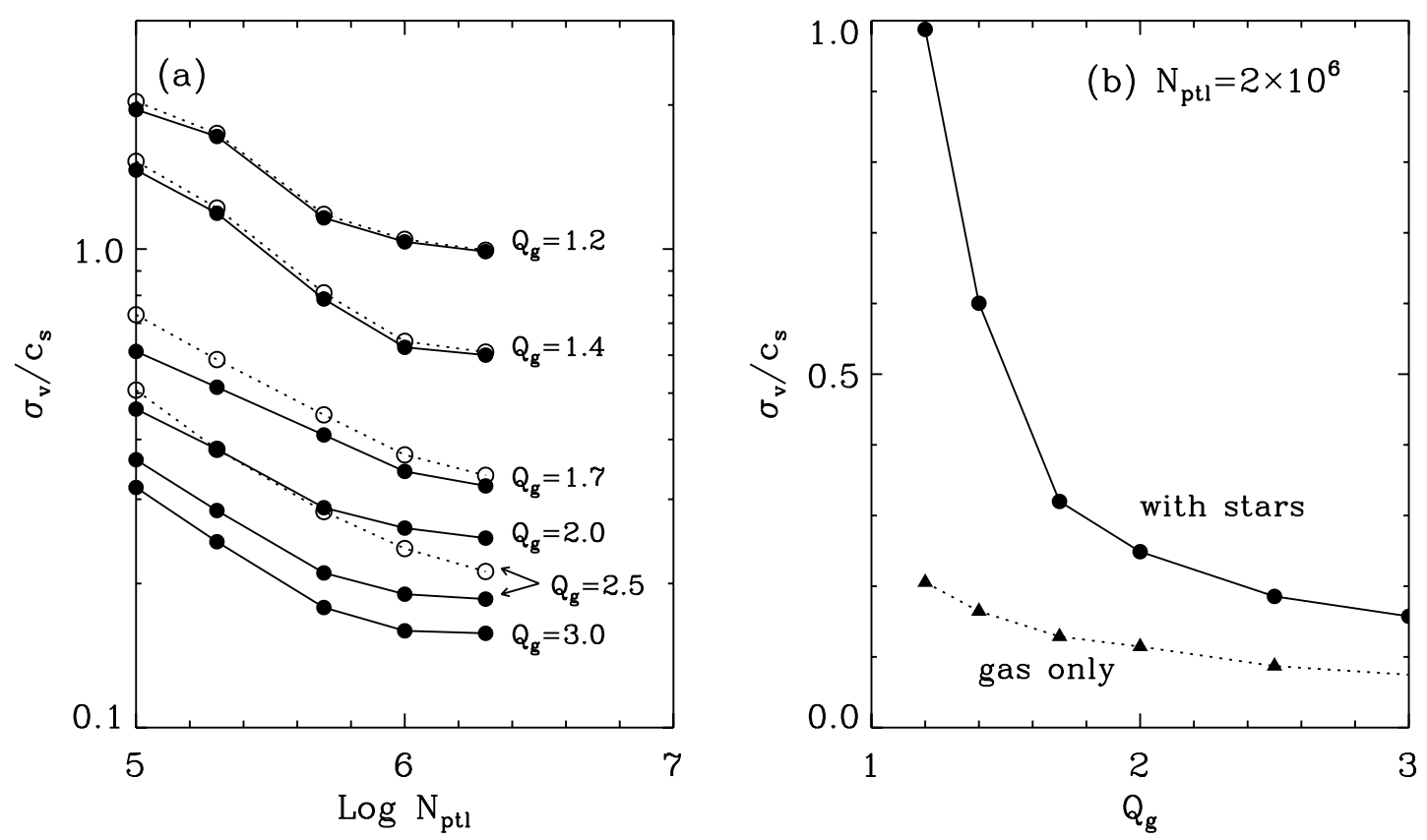

Fig. 12. - Amplitudes of mean fluctuating gaseous velocities $\sigma_{v}$ in two-component models with $Q_{s}=2.1, H_{g}=0.87 c_{g} / \kappa$, and $H_{s}=0.4 \sigma_{x} / \kappa$, to test numerical resolution effects. (a) Filled circles show $\sigma_{v}$ as a function of stellar particle number $N_{\mathrm{ptl}}$ after subtracting the random kinetic energy induced by Poisson noise in the stellar distribution, while open circles give $\tilde{\sigma}_{v}$, the gas velocity dispersion without this correction. (b) Filled circles give $\sigma_{v}$ for $N_{\mathrm{ptl}}=2 \times 10^{6}$, and filled triangles are for gas-only systems. See text for details.

Even after $\delta E_{\text {kin }}^{\mathrm{NG}}$, embodying the kinetic energy directly induced in the gaseous disk by the stellar Poisson noise, has been subtracted, Figure 12 shows that $\sigma_{v}$ still depends on $N_{\mathrm{ptl}}$. This is because the gas responds to swing-amplified perturbations in the stars that partly originate as noise in the initial stellar distribution. Note, however, that $\sigma_{v}$ clearly converges with increasing $N_{\text {ptl }}$, im- plying that this secondary noise effect becomes negligible. The convergence study suggests that $\sigma_{v}$ at $N_{\mathrm{ptl}}=2 \times 10^{6}$ provides a clean measure of the turbulent energy that is uncontaminated by effects of Poisson noise. Our results also imply that numerical simulations of marginally unstable galactic disks are not reliable if insufficiently many particles are employed for the collisionless part.
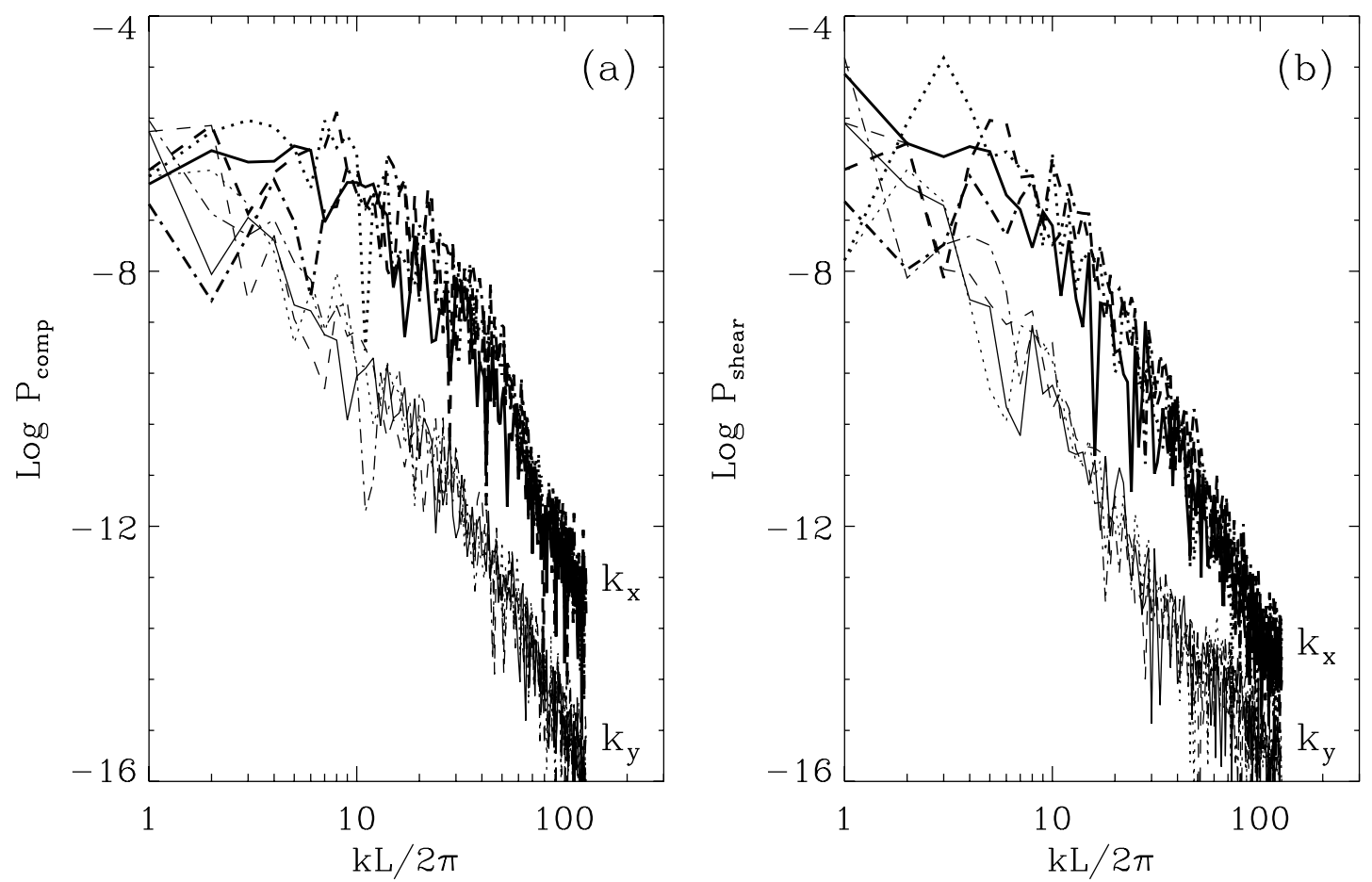

Fig. 13. - Amplitudes of the power spectra along the $k_{x}$ - and $k_{y}$-axes of $(a)$ compressive and $(b)$ shearing parts of the gaseous velocity in a two-component model with $Q_{g}=1.4$ at $t / t_{\mathrm{orb}}=2.5$. Solid, dotted, dashed, and dot-dashed lines indicate the modes with $k_{y} L /(2 \pi)=0,1,2,3$ as a function of $k_{x}$ (thick lines) and the modes with $k_{x} L /(2 \pi)=0,1,2,3$ as a function of $k_{y}$ (thin lines), respectively. The velocity power is clearly anisotropic, and the shearing part dominates at large scale. Both compressive and shearing parts along the $k_{x}$-axis show a break near $k_{x} L /(2 \pi) \sim 8-10$ below which the power flattens. 
Figure $12 b$ plots $\sigma_{v}$ for two-component disks with $N_{\mathrm{ptl}}=2 \times$ $10^{6}$ as well as the velocity dispersion for gas-only disks. For stable systems with $Q_{g} \gtrsim 2.0$, the stellar component enhances the amplitudes of the gaseous random velocities by about a factor of 2 compared to the gas-only counterpart, although velocity dispersions are small overall in this regime. When the disk is marginal or unstable $\left(Q_{g}=1.2\right.$ or 1.4$), \sigma_{v}$ becomes comparable to the effective speed of sound, and is $\sim 4-5$ times larger than the value obtained for a gas-only disk. This suggests not only that the stellar disk can significantly affect driving of gaseous turbulence, but also that self-gravity combined with galactic shear is capable of inducing sonic-level gaseous turbulence under realistic disk conditions.

To characterize the turbulence generated by swing amplification, we plot in Figure 13 Fourier power spectra of the compressive and shear components of gaseous velocities defined by

$$
P_{\text {comp }}=\left|\hat{\boldsymbol{k}} \cdot \delta \boldsymbol{v}_{k}\right|^{2}, \quad P_{\text {shear }}=\left|\hat{\boldsymbol{k}} \times \delta \boldsymbol{v}_{k}\right|^{2}
$$

respectively, where $\delta \boldsymbol{v}_{k}$ denotes the Fourier transform of the perturbed velocity, $\boldsymbol{v}-\boldsymbol{v}_{0}$. Data at $t / t_{\text {orb }}=2.5$ of a fully gravitating two-component model with $Q_{g}=1.4$ and $N_{\text {ptl }}=2 \times 10^{6}$ are used for Figure 13. We have also calculated the density power spectra and confirmed that they follow the compressive velocity power spectra very closely.

The velocity power spectra are clearly anisotropic, as is typical of turbulence in systems with strong background shear (e.g., Hawley et al. 1995; Kim et al. 2003). Comparisons of Figures $13 a$ and $13 b$ reveal that the ratio of total power in the shearing to compressive parts is about 1.5 . This value is quite small compared to $\sim 4-10$ found in recent simulations of hypersonic turbulence (e.g., Boldyrev et al. 2002; Vestuto et al. 2003), presumably reflecting the facts that (1) our models are subsonic or weakly supersonic at best, so that compressions are not strongly dissipated, and (2) driving by self-gravity favors pumping of compressive motions. Although the power spectra vary too much at low $k$ to yield clean power-law indices and are affected by numerical dissipation at high $k$ (e.g., Kim \& Ryu 2005), our results are consistent with $P \propto k_{y}^{-4.5}$ in the inertial range. The cuts along the $k_{x}$-axis exhibit a break near $k_{x} L /(2 \pi) \sim 8-10$, below which the power flattens and above which $P_{\text {comp }} \propto k_{x}^{-4}$ and $P_{\text {shear }} \propto k_{x}^{-6}$. About $85 \%$ of the turbulent power is in large-scale modes with $\lambda \geq L / 10$, suggesting that the small-scale turbulent velocity dispersion is a small fraction of the total; this proportion could, however, differ in three-dimensional models. The flattening of power spectra at small $k_{x}$ and steepening at large $k_{x}$ in our models makes sense, considering that in turbulence driven predominantly by swing amplification the background shear tends to increase the $k_{x}$ value of any structure over time. In quasi-steady state, nonlinear interactions of shearing wavelets evidently supply fresh power into small- $\left|k_{x}\right|$ modes at just the rate needed to compensate for the kinematic shift of $k_{x}$ due to shear, such that the shape of the power spectrum is nearly flat. While turbulent energy cascades to small scales appear to shape power spectra along the $k_{y}$-direction, shear and nonlinear feedback clearly dominate energy flows in the $k_{x}$-direction.

\section{SUMMARY AND DISCUSSION}

\subsection{Summary}

In this paper we have analyzed the nonlinear dynamical evolution of two-component galactic disks. One primary goal has been to extend our previous work (Paper I), which modeled gas-only disks, to evaluate thresholds for runaway gravitational instability and formation of bound clouds. The other primary goal has been to quantify the generation of gaseous turbulence in cases where gravitational runaway does not occur. The gaseous medium is isothermal and evolved by a hydrodynamic technique as in Paper I, whereas the newly implemented stellar component is represented by an $N$-body system evolved by a particle-mesh method. The two components interact through their respective gravitational potentials. Our simulations are two-dimensional, but incorporate (important) finite disk thickness effects in an approximate fashion (see $\S 2.1$ ). The local framework we employ incorporates galactic rotational shear, tidal gravity, and Coriolis forces. The present models do not include effects of magnetic fields or externally driven stellar density waves.

Our main results are as follows.

1. Allowing for nonzero disk thickness significantly stabilizes axisymmetric modes in two-component disks (see $\S 3$ ). For solar neighborhood conditions with $Q_{s}=2.1$, the critical Toomre parameter of the gaseous component would be $Q_{g \text {,crit }}=1.27$ if both the disks were razor thin. Using more realistic scale heights $H_{g}=$ $170 \mathrm{pc}$ and $H_{s}=330 \mathrm{pc}$ for the gaseous and stellar disks, respectively, the critical value is reduced to $Q_{g \text {,crit }}=0.67$, well below the observed value. Two-component thick disks with $Q_{g}=1.4$ are axisymmetrically stable unless $Q_{s}<0.8$, which is again inconsistent with observed galactic conditions. Star formation thresholds at $Q_{g \text {,th }} \sim 1.4$ observed in the outer regions of external massive disk galaxies (Kennicutt 1989; Martin \& Kennicutt 2001) are therefore not a consequence of axisymmetric gravitational instability.

2. Our two-dimensional simulations show that two-component disks undergo gravitational runway when $Q_{g}<Q_{g \text {,crit }}(\operatorname{see} \S 5.1)$. For stellar parameters representing the solar neighborhood $\left(Q_{s}=\right.$ 2.1 and $H_{s}=330 \mathrm{pc}$ ), this nonlinear threshold occurs at $Q_{g, \text { crit }} \sim$ 1.4. Disks with $Q_{g}$ below this threshold experience nonaxisymmetric gravitational instability, forming bound condensations of mass $\sim 6 \times 10^{7} M_{\odot}$ each. These condensation masses are 10 times larger than those that form in razor-thin, gas-only disks, because larger scales are required for gravitational instability in thicker disks. We note that the critical value of $Q_{g}$ for nonaxisymmetric instability is larger than that for axisymmetric instability by more than a factor of 2 .

3. In addition to forming bound condensations, swing amplification is able to generate a significant level of gaseous turbulence (see $\S 5.2$ ). The active stellar component is important to this, with velocity dispersions a factor $2-5$ larger than in the equivalent gasonly disks. Our simulation results show that when $Q_{g}=1.2-1.6$, corresponding to disks near the stability threshold, the densityweighted velocity dispersions of the gas amount to $\sigma_{v} \sim(0.3-1) c_{g}$. This suggests that swing amplification can serve to tap rotational and gravitational energy in feeding random motions in the ISM. The turbulence in our models is anisotropic and has slightly more energy in the shearing than the compressive motions. Below $k_{x} L /$ $(2 \pi) \lesssim 8-10$, the power spectrum is relatively flat, while it steepens at larger $k$. Most $(\sim 85 \%)$ of the total power is contained in large-scale modes with $\lambda \geq L / 10$, although in fully threedimensional models the relative power in small and large scales could potentially change.

4. Poisson noise in the positions of randomly placed particles produces initial density fluctuation amplitudes $\delta \Sigma / \Sigma \approx 1.4\left(N_{\mathrm{ptl}} /\right.$ $\left.10^{4}\right)^{-1 / 2}$ for two-dimensional particle-mesh simulations with grid resolution $256^{2}$. For near-marginal systems we show that these density variations can lead to spurious local instabilities and artificial fragmentation $(\S 4.2)$. Poisson noise can also lead to overestimates of turbulent velocity fluctuations produced by swing. 
Insufficient particle numbers can therefore seriously compromise the results of $N$-body disk simulations. Care must be taken to test numerical convergence. Here, we find that $N_{\text {ptl }}=1-2 \times 10^{6}$ is needed to avoid contamination by Poisson noise.

\subsection{Discussion}

In this work we have found that the threshold for formation of gravitationally bound gas clouds occurs at $Q_{g \text {,crit }} \sim 1.4$. This result is in fact nearly identical to the nonlinear threshold value $Q_{g \text {,crit }}=1.3$ we found in Paper I (for unmagnetized models), due to compensation between two effects that were not accounted for in Paper I. The current models allow for a (stabilizing) nonzero disk thickness and include a (destabilizing) active stellar component; although each effect by itself makes a significant difference in $Q_{g, \text { crit }}$, in net they nearly cancel each other. While the present models do not include magnetic fields, we expect that inclusion of magnetic effects would not significantly change the results; in $\mathrm{Pa}$ per I, there was only a $10 \%$ change in $Q_{g \text {,crit }}$ even with equipartitionstrength large-scale magnetic fields.

An open question is how sensitive $Q_{g \text {,crit }}$ is to the precise parameters characterizing the stellar disk. To define a manageable parameter space, we have fixed the value of $Q_{s}$ to 2.1, the (initial) ratio of the stellar velocity dispersion to the gas sound speed to 4.3, and the ratio of stellar to gas disk thicknesses to 1.9. The values chosen for these parameters are based on solar neighborhood observations and, thus, are representative of conditions in the middle of the Galactic disk for a late-type spiral. Although the stellar component's parameters surely vary from the deep interior to the outer reaches of a galactic disk, constraining the relevant quantities observationally is in fact quite challenging. Extragalactic studies (van der Kruit \& Searle 1981, 1982; de Grijs \& Peletier 1997) are consistent with the stellar disk thicknesses being independent of radius at least for late-type spirals. In this case, a (self-gravitating) stellar disk with a constant ratio of vertical to radial velocity dispersion will have $\sigma_{x} \propto \Sigma_{s 0}^{1 / 2}$. For an exponential stellar surface density $\Sigma_{s 0} \propto \exp \left(-R / R_{d}\right)$ with scale length $R_{d}$, this would imply that the variation of $Q_{s}$ over $1<R / R_{d}<4$ is less than $30 \%$. Furthermore, $Q_{s}$ varies over only a small range from one galaxy to another; Bottema (1993) found that $Q_{s}=2-2.5$ fits the data well (consistent with theoretical predictions of, e.g., Sellwood \& Carlberg 1984). Thus, although we obtained the result $Q_{g \text {,crit }} \sim 1.4$ for a specific set of stellar parameters, the modest variation in these parameters among and within real galaxies suggests that there would be a correspondingly modest variation in $Q_{g \text {,crit }}$.

It is interesting to note that measured values of $Q_{g}$ at the observed threshold radii for star formation are around $Q_{g \text {,th }} \sim 1-2$ (Kennicutt 1989; Martin \& Kennicutt 2001). Unless the stellar properties at these threshold radii differ dramatically from those we have adopted, the results of our work, showing $Q_{g \text {,crit }}$ within the same range, quantitatively support the possibility that star formation thresholds and (nonaxisymmetric) gravitational instability thresholds are likely to be one and the same. A similar conclusion was reached very recently by $\mathrm{Li}$ et al. (2005) who performed global SPH $+N$-body simulations and found that a critical "combined star-gas" Toomre parameter of $Q_{\text {sg }}=1.6$ defines the limit between gravitationally stable and unstable disks. Of course, turbulence driven by swing amplification could change the effective value of $Q_{q}$ if it contributes a stabilizing pressure in a manner similar to the microphysical random motions and if the turbulent amplitudes are large. Since the threshold radii are by definition where disks become stable, however, then $Q_{g}>Q_{g, \text { crit }}$ in those locations, and there would not be a significant contribution from swing-driven turbulence.
We note that many physical processes not included in the current models may potentially change $Q_{g \text {,crit }}$ for gravitational runaway. These include turbulence pervasive in the ISM and thermal and other dynamical instabilities. For instance, Kim et al. (2003) found that nonlinear density fluctuations driven by magnetorotational instability in gas-only, three-dimensional disks enhance $Q_{g \text {,crit }}$ by about $50 \%$ relative to those in unmagnetized counterparts. It is especially important to explore the effects of turbulent motions and magnetic fields in multiphase systems, because the thermal velocity dispersion of cold gas is so low. The usual assumption is that an effective $Q$ can be defined by appropriate weighting of various contributing effective pressures and surface density components (e.g., Wada et al. 2002); an important direction for future research is to test this idea rigorously. ${ }^{6}$ In future work, it will be interesting to test whether realistic turbulent, multiphase models (in three dimensions or with thick-disk two-dimensional gravity) show nonlinear threshold behavior for a suitably defined $Q_{g \text {,eff }}$ and how a live stellar component quantitatively affects critical $Q$ values.

The fact that the stellar disk plays a significant role in destabilizing the gas disk may help explain the very low star formation rates in late-type, low surface brightness (LSB) galaxies. While LSB galaxies are often comparable in total gas mass to normal galaxies (e.g., Matthews et al. 2005), most of them have gas surface densities below the threshold values $\Sigma_{g \text {,th }}$ corresponding to $Q_{g}=$ 1.4 , such that low star formation rates and weak stellar disks are easily explained (e.g., van der Hulst et al. 1993; de Blok et al. 1996; Uson \& Matthews 2003). However, some LSB galaxies with $Q_{g}<1.4$ still evidence little star formation (Pickering et al. 1997, 1999; O’Neil et al. 2000a, 2000b; see also Elmegreen 2002). In part, this is probably because $Q_{s}$ is so large that the stellar disk does little to encourage instability in the gas disk; we found that pure-gas disks are still quite stable when $Q_{g}=1.2$. In addition, without the vertical gravity of a massive stellar disk, the gas disk thickness will increase by a factor of 1.5-2 (e.g., Kim et al. 2002) compared to that in a normal galaxy. A larger value of $H_{g}$ dilutes gravity, which tends to further lower $Q_{g \text {,crit. }}$ Together, these effects will reduce $Q_{g \text {,crit }}$ below 1 for LSB galaxies, making them more stable than previously thought.

Galactic differential rotation represents a bountiful store of kinetic energy (e.g., von Weizsäcker 1951), and we have shown that swing amplification in two-component disks is able to transform this energy into ISM turbulent motions with appreciable amplitudes. Other mechanisms that can channel sheared rotation into turbulence in the diffuse ISM include the magnetorotational instability (Sellwood \& Balbus 1999; Kim et al. 2003; Piontek \& Ostriker 2004, 2005) and interactions with spiral arms (Martos \& Cox 1998; Gómez \& Cox 2004; Kim \& Ostriker 2006; Kim et al. 2006). Our current simulations show that provided that the disk is marginal or unstable, swing amplification is at least as efficient as the magnetorotational instability under conditions of comparable surface density. Two-phase self-gravitating hydrodynamic models ( Wada et al. 2002) and self-gravitating dissipative cloudfluid models (Huber \& Pfenniger 2001) have similarly concluded that self-gravity coupled with galactic rotation can produce ISM turbulence that is sustained over many galactic rotations.

Driving of turbulence at levels $\sigma_{v} \gtrsim 0.6 c_{g}$ requires a disk to be at least marginally unstable. Because the outer, low surface

\footnotetext{
6 Single-phase models such as the present one are not useful for evaluating the idea or quantifying the value of $Q_{g \text {, eff }}$, since naive inclusion of the radial turbulent velocities that are present $\left(<0.5 c_{g}\right)$ at both small and large scales increases $Q_{g}$ by $<10 \%$.
} 
density regions of disks are quite stable, this mechanism cannot drive turbulence there. ${ }^{7}$ But in the inner parts of disks, selfgravitational stirring in some regions (having slightly lower density) may go hand-in-hand with the formation of massive, starforming clouds in other regions (having slightly higher density). Because the fraction of gas converted to stars per cloud formation epoch is quite small $(\lesssim 10 \%)$, the rate of reduction in the mean gas surface density — and hence rate of increase in $Q_{g}$-is very low. As $Q_{g}$ increases over time, the timescale for cloud formation via

\footnotetext{
${ }^{7}$ The outer parts of disks, however, are also where the scale height flares; together with low $\mathrm{H}$ I surface density this implies low gas volume densities, which is most favorable for the magnetorotational instability to develop (Piontek \& Ostriker 2007).
}

gravitational instability also increases. The overall evolution is toward an asymptotic state in which $Q_{g}$ approaches $Q_{g, \text { crit }}$. Galaxies may naturally "fine-tune" their parameters in such a way that self-gravity can help stabilize small scales - by generating turbulence-even as it forces large scales into collapse.

W.-T. K. gratefully acknowledges the assistance and hospitality provided by the Department of Astronomy at the University of Maryland while this paper was prepared. W.-T. K. is supported by Korea Science and Engineering Foundation (KOSEF) grant R01-2004-000-10490-0 at Seoul National University. This work is partially supported by NASA grant NNG $05-$ GG43G to the University of Maryland.
Balbus, S. A., \& Cowie, L. L. 1985, ApJ, 297, 61

Barbanis, B., \& Woltjer, L. 1967, ApJ, 150, 461

Bertin, G., \& Lin, C. C. 1996, Spiral Structure in Galaxies: A Density Wave Theory (Cambridge: MIT Press)

Bertin, G., \& Romeo, A. B. 1988, A\&A, 195, 105

Binney, J., \& Tremaine, S. 1987, Galactic Dynamics (Princeton: Princeton Univ. Press)

Boldyrev, S., Nordlund, Å., \& Padoan, P. 2002, ApJ, 573, 678

Bottema, R. 1993, A\&A, 275, 16

Boulares, A., \& Cox, D. P. 1990, ApJ, 365, 544

Caldwell, N., Kennicutt, R., Phillips, A. C., \& Schommer, R. A. 1991, ApJ, 370,526

Carlberg, R. G., \& Sellwood, J. A. 1985, ApJ, 292, 79

Chen, B., et al. 2001, ApJ, 553, 184

de Blok, W. J. G., McGaugh, S. S., \& van der Hulst, J. M. 1996, MNRAS, 283, 18

de Grijs, R., \& Peletier, R. F. 1997, A\&A, 320, L21

De Simone, R., Wu, X., \& Tremaine, S. 2004, MNRAS, 350, 627

Dickey, J. M., Hanson, M. M., \& Helou, G. 1990, ApJ, 352, 522

Dickey, J. M., \& Lockman, F. J. 1990, ARA\&A, 28, 215

Elmegreen, B. G. 1987, ApJ, 312, 626

. 1995a, in The 7th Guo Shoujing Summer School on Astrophysics:

Molecular Clouds and Star Formation, ed. C. Yuan \& H. You (Singapore:

World Scientific), 149 1995b, MNRAS, 275, 944

2002, ApJ, 577, 206

Elmegreen, B. G., \& Elmegreen, D. M. 1983, MNRAS, 203, 31

Elmegreen, B. G., Elmegreen, D. M., \& Leitner, S. N. 2003, ApJ, 590, 271

Elmegreen, B. G., \& Scalo, J. 2004, ARA\&A, 42, 211

Fuchs, B. 2001, MNRAS, 325, 1637

Fuchs, B., Dettbarn, C., \& Tsuchiya, T. 2005, A\&A, 444, 1

Gammie, C. F. 1996, ApJ, 462, 725 2001, ApJ, 553, 174

Goldreich, P., \& Lynden-Bell, D. 1965a, MNRAS, 130, 97 1965b, MNRAS, 130,125

Gómez, G. C., \& Cox, D. P. 2004, ApJ, 615, 744

Griv, E., Rosenstein, B., Gedalin, M., \& Eichler, D. 1999, A\&A, 347, 821

Hawley, J. F., Gammie, C. F., \& Balbus, S. A. 1995, ApJ, 440, 742

Heiles, C. 2001, in ASP Conf. Ser. 231, Tetons 4: Galactic Structure, Stars, and the Interstellar Medium, ed. C. E. Woodward, M. D. Bicay, \& J. M. Shull (San Francisco: ASP), 294

Hockney, R. W., \& Eastwood, J. W. 1988, Computer Simulation Using Particles (Philadelphia: Adam Hilger)

Holmberg, J., \& Flynn, C. 2000, MNRAS, 313, 209

Huber, D., \& Pfenniger, D. 2001, A\&A, 374, 465

Hunter, C. 1964, ApJ, 139, 570

Jog, C. J. 1992, ApJ, 390, 378

1996, MNRAS, 278, 209

Jog, C. J., \& Solomon, P. M. 1984a, ApJ, 276, 114 1984b, ApJ, 276, 127

Julian, W. H., \& Toomre, A. 1966, ApJ, 146, 810

Karaali, S., Bilir, S., \& Hamzaoğlu, E. 2004, MNRAS, 355, 307

Kennicutt, R. C. 1989, ApJ, 344, 685

\section{REFERENCES}

Kennicutt, R. C. 1997, in The Interstellar Medium in Galaxies, ed. J. M. van der Hulst (Dordrecht: Kluwer), 171

Kim, C.-G., Kim, W.-T., \& Ostriker, E. C. 2006, ApJ, 649, L13

Kim, J., \& Ryu, D. 2005, ApJ, 630, L45

Kim, W.-T., \& Ostriker, E. C. 2001, ApJ, 559, 70 (Paper I) 2006, ApJ, 646, 213

Kim, W.-T., Ostriker, E. C., \& Stone, J. M. 2002, ApJ, 581, 1080

2003, ApJ, 599, 1157

Knapen, J. H., Cepa, J., Beckman, J. E., Soledad del Rio, M., \& Pedlar, A. 1993, ApJ, 416, 563

Kuijken, K., \& Gilmore, G. 1989, MNRAS, 239, 605

Li, Y., Mac Low, M.-M., \& Klessen, R. S. 2005, ApJ, 620, L19

. 2006, ApJ, 639, 879

Lin, C. C., \& Shu, F. H. 1966, Proc. Natl. Acad. Sci., 55, 229

Lin, C. C., Yuan, C., \& Shu, F. H. 1969, ApJ, 155, 721

Lubow, S. H., Balbus, S. A., \& Cowie, L. L. 1986, ApJ, 309, 496

Mac Low, M.-M., \& Klessen, R. S. 2004, Rev. Mod. Phys., 76, 125

Martin, C. L., \& Kennicutt, R. C. 2001, ApJ, 555, 301

Martos, M. A., \& Cox, D. P. 1998, ApJ, 509, 703

Matthews, L. D., Gao, Y., Uson, J. M., \& Combes, F. 2005, AJ, 129, 1849

Minchev, I., \& Quillen, A. C. 2006, MNRAS, 368, 623

Monaghan, J. J. 1989, J. Comput. Phys., 82, 1

O’Neil, K., Bothun, G. D., \& Schombert, J. 2000a, AJ, 119, 136

O'Neil, K., Verheijen, M. A. W., McGaugh, S. S. 2000b, AJ, 119, 2154

Pickering, T. E., Impey, C. D., van Gorkom, J. H., \& Bothun, G. D. 1997, AJ, 114,1858

Pickering, T. E., van Gorkom, J. H., Impey, C. D., \& Quillen, A. C. 1999, AJ, 118,765

Piontek, R. A., \& Ostriker, E. C. 2004, ApJ, 601, 905 2005, ApJ, 629, 849 2007, ApJ, in press

Quirk, W. J. 1972, ApJ, 176, L9

Rafikov, R. R. 2001, MNRAS, 323, 445

Romeo, A. B. 1992, MNRAS, 256, 307

Rybicki, G. B. 1971, Ap\&SS, 14, 15

Sellwood, J. A., \& Balbus, S. A. 1999, ApJ, 511, 660

Sellwood, J. A., \& Carlberg, R. G. 1984, ApJ, 282, 61

Stone, J. M., \& Norman, M. L. 1992, ApJS, 80, 753

Toomre, A. 1964, ApJ, 139, 1217

. 1981, in Structure and Evolution of Normal Galaxies, ed. S. M. Fall \& D. Lynden-Bell (Cambridge: Cambridge Univ. Press), 111

Uson, J. M., \& Matthews, L. D. 2003, AJ, 125, 2455

van der Hulst, J. M., Skillman, E. D., Smith, T. R., Bothun, G. D., McGaugh, S. S., $\&$ de Blok, W. J. G. 1993, AJ, 106, 548

van der Kruit, P. C., \& Searle, L. 1981, A\&A, 95, 105 1982, A\&A, 110, 61

van Zee, L., \& Bryant, J. 1999, AJ, 118, 2172

Vestuto, J. G., Ostriker, E. C., \& Stone, J. M. 2003, ApJ, 590, 858

von Weizsäcker, C. F. 1951, ApJ, 114, 165

Wada, K., Meurer, G., \& Norman, C. A. 2002, ApJ, 577, 197

White, R. L. 1988, ApJ, 330, 26

Wisdom, J., \& Tremaine, S. 1988, AJ, 95, 925

Wong, T., \& Blitz, L. 2002, ApJ, 569, 157 